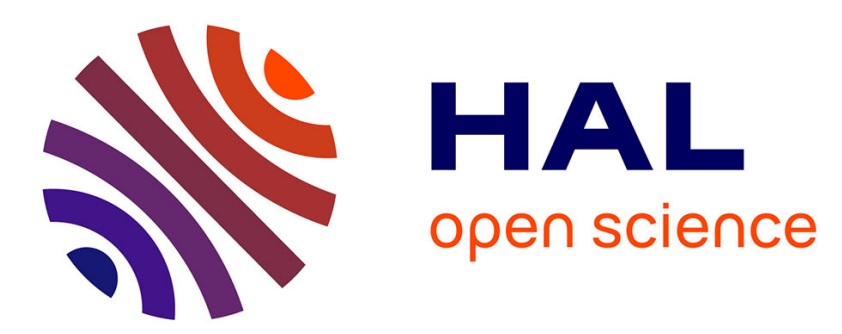

\title{
De la modélisation des situations de travail à la caractérisation des compétences: Une approche par logique floue.
}

Farouk Belkadi, Eric Bonjour, Maryvonne Dulmet

\section{- To cite this version:}

Farouk Belkadi, Eric Bonjour, Maryvonne Dulmet. De la modélisation des situations de travail à la caractérisation des compétences: Une approche par logique floue.. Journal Européen des Systèmes Automatisés (JESA), 2009, 43 (1-2), pp.35-71. 10.3166/jesa.43.35-71 . hal-00434059

\author{
HAL Id: hal-00434059 \\ https://hal.science/hal-00434059
}

Submitted on 20 Nov 2009

HAL is a multi-disciplinary open access archive for the deposit and dissemination of scientific research documents, whether they are published or not. The documents may come from teaching and research institutions in France or abroad, or from public or private research centers.
L'archive ouverte pluridisciplinaire HAL, est destinée au dépôt et à la diffusion de documents scientifiques de niveau recherche, publiés ou non, émanant des établissements d'enseignement et de recherche français ou étrangers, des laboratoires publics ou privés. 


\title{
De la modélisation des situations de travail à la caractérisation des compétences : Une approche par la logique floue
}

\author{
Farouk Belkadi* — Eric Bonjour** - Maryvonne Dulmet** \\ * Université de Technologie de Compiègne - CNRS UMR 6253 - Roberval \\ BP 60319, rue du Docteur Schweitzer, 60203 Compiègne Cedex, France \\ e-mail : fbelkadi@utc.fr \\ ** Institut FEMTO-ST, UMR CNRS 6174 - UFC / ENSMM / UTBM, \\ Département Automatique et Systèmes Micro-Mécatroniques (AS2M), \\ 24, rue Alain Savary, 25000 Besançon, France. \\ e-mail : ebonjour@ens2m.fr ; mdulmet@ens2m.fr
}

RÉSUMÉ. Face à un contexte technologique et organisationnel de plus en plus évolutif, les managers ont besoin d'outils pour les aider à améliorer le pilotage des compétences mobilisées dans les activités de conception. Notre contribution vise à fournir une aide à la caractérisation des compétences considérée comme une première étape dans le processus de pilotage des compétences. La démarche de caractérisation proposée s'effectue en deux phases. La première phase s'appuie sur une qualification des différentes caractéristiques de la situation de travail. La deuxième est réalisée par un système à inférences floues qui convertit le résultat de cette "qualification" en valeur estimée des composants de la compétence associée. L'application de la méthode en milieu industriel nous a permis de confirmer la faisabilité de ce "principe de solution" pour une aide à la caractérisation dynamique des compétences.

ABSTRACT. To cope with the fast change of the technological and organizational context, design managers need tools to help them to improve competence management. Our contribution aims at supporting the task of competence characterization that is considered as the first step of the competence management process. The proposed characterization approach involves two stages. The first stage concerns the qualifications of the working situation characteristics. The second stage is realized by a fuzzy logic system which converts the result of the step of "qualification" into an estimated value of the components of the associated competence. The application of the method in an industrial case confirms the possibility of using this "principle of solution" to provide a dynamic characterization of competencies.

MOTS-CLÉS : Caractérisation des compétences, traçabilité, modèle de situation, logique floue. KEYWORDS: Competence characterization, traceability, situation model, fuzzy logic 


\section{Introduction}

La recherche de performance dans un contexte évolutif impose à l'entreprise d'avoir plus de flexibilité dans son organisation de travail, une meilleure maîtrise des technologies émergeantes mais surtout une meilleure considération de ses ressources humaines. Au début de la révolution industrielle, les entreprises ont parié sur la technologie comme vecteur de progrès et de performance (Midler, 1997), (Jolis, 1997). Dans le passé, les organisations traitaient la ressource humaine au même titre que les autres ressources matérielles. Aujourd'hui, tous les acteurs, industriels et scientifiques, s'accordent à dire que l'obtention de la performance ne peut se faire sans l'implication de l'ensemble des acteurs de l'entreprise, détenteurs de connaissances et développant leurs compétences individuelles et collectives collectives (Spencer et al., 1993), (LeBoterf, 2000), (Dejoux 2001).

Le domaine de la conception est l'un des domaines les plus concernés par ces nouvelles évolutions (Sardas, 1997) en raison de la complexité croissante des activités de conception, de la position centrale que joue la conception dans la chaîne de création de valeur et du caractère fortement cognitif et collectif de ces activités (Micaëlli et al., 2003).

Les travaux concernant l'ingénierie et la gestion des connaissances, développés ces dernières décennies, sont une conséquence de cette prise de conscience relative au rôle incontournable des ressources humaines dans les organisations. Nonaka et Takeuchi (Nonaka et al., 1995) sont parmi les premiers à souligner l'importance de sauvegarder le réservoir des connaissances dans une organisation. C'est ce que Grundstein (Grundstein, 2001) a qualifié de capital immatériel devant être repéré, préservé, valorisé et actualisé en permanence pour garantir la pérennité des savoirfaire critiques des experts et pour les partager entre les acteurs.

Le concept de compétence a été introduit pour mieux valoriser le capital immatériel. Ce concept a remplacé la vision classique de la qualification et s'est imposé comme un facteur incontournable de la performance (Jolis, 1997). Il a permis une meilleure intégration du potentiel humain dans la définition des stratégies de l'entreprise. Dans les années 90, Prahalad et Hamel (Prahalad et al., 1990) ont montré qu'il était désormais nécessaire pour les entreprises de se considérer comme des "productrices de compétences distinctives" et qu'elles devaient veiller au développement et à la pérennité de ces compétences.

La caractérisation de compétences est l'un des processus fondamentaux dans le pilotage des compétences. Elle permet de définir la compétence à travers des éléments formalisables, évaluables et stockables dans des référentiels (Acuna et al., 2004), (Harzallah et al., 2006). En raison de l'évolution rapide des missions et des situations de travail, les compétences tendent à évoluer rapidement et les référentiels deviennent difficiles à mettre à jour.

Les méthodes de caractérisation des compétences sont généralement empiriques et émanent principalement du domaine de GRH pour la construction des référentiels 
(Rault-Jacquot, 1993). Elles reposent sur une analyse des situations de travail a posteriori. Cette analyse, relativement subjective, est obtenue à partir d'une observation du déroulement des activités, de l'auto-description du poste ou encore, de l'analyse des incidents critiques survenus durant l'activité.

Afin de réduire les limites constatées sur les démarches empiriques, nous proposons dans cet article, une nouvelle méthode visant à automatiser les différentes étapes du processus de caractérisation des compétences. L'objectif est de donner plus d'objectivité aux données descriptives de la situation de travail et celles relatives aux caractéristiques des compétences. Par rapport au référentiel de compétence, cela se traduit par une évaluation, relative à une classe de situation spécifique, du niveau de compétence validé chez un acteur et pouvant être requis pour réaliser une mission semblable. Pour cela, nous proposons d'intégrer trois outils complémentaires: une méthode de traçabilité pour recueillir les informations de la situation de travail, des outils mathématiques pour évaluer ses caractéristiques et un système à inférences floues pour représenter le raisonnement de l'expert "manager".

Dans la section suivante, nous revenons sur les concepts de connaissance et compétence, leurs définitions dans la littérature, la problématique de traçabilité et de gestion des connaissances, les différents axes de pilotage des compétences ainsi que l'intérêt de la caractérisation pour le pilotage. Dans la section 3, nous présentons les hypothèses de base et le principe général de l'approche proposée.

Le domaine d'application de nos travaux concerne les activités de conception. Dans les sections 4, 5 et 6 , nous développons progressivement les différents modules de notre démarche, pour la caractérisation des compétences dans le cadre d'un projet de conception. Enfin, dans la section 7, nous présentons un exemple d'utilisation de la méthode dans un milieu industriel.

\section{Connaissance et compétence dans la littérature}

Le concept de compétence est intrinsèquement lié aux concepts de connaissance (Berio et al., 2005), (Prax 2000), de savoir et de savoir-faire (Harzallah, 2000). Dans ce qui suit nous présentons une revue de littérature sur les domaines de gestion de connaissances et pilotage des compétences. Notre approche repose sur une articulation entre ces deux dimensions.

\subsection{Définitions}

\subsubsection{Définition de "la connaissance"}

Les chercheurs en ingénierie des connaissances et en génie industriel semblent converger aujourd'hui sur une caractéristique clé de la connaissance : une connaissance n'existe pas en dehors d'un individu, elle est de l'ordre d'une 
représentation cognitive (Ganascia, 1996). Pour (Prax, 2000), "la connaissance résulte d'une acquisition d'information et d'une action, elle est à la fois mémoire et processus de construction d'une représentation". Des auteurs, comme (Skyrme, 1994), soulignent aussi que la spécificité de la connaissance est qu'elle est dépendante d'une activité cognitive humaine. C'est une combinaison de sens du contexte, de mémoire personnelle et de processus cognitif. Cette définition rejoint celle proposée dans (Ermine 1996) où la connaissance est perçue comme de "l'information qui prend une certaine signification dans un contexte donné".

Pour (Nonaka et al., 1995), "la connaissance est une croyance vraie et justifiée par le contexte, elle attribue la croyance vraie à un individu ou à une communauté". Dans leur modèle de création et transfert des connaissances, les auteurs distinguent deux catégories de connaissances : les connaissances explicites (ou explicitables) sont les connaissances pouvant être facilement extraites et représentées sur des supports partageables. A l'inverse, les connaissances tacites sont difficilement explicitables. Ces deux catégories sont associées respectivement aux concepts de savoir et de savoir faire (Grundstein, 2001).

\subsubsection{Définitions de "la compétence"}

La définition de la compétence a été abordée par plusieurs champs disciplinaires. Le Boterf (Le Boterf, 2002) définit une personne compétente comme "une personne qui sait agir avec pertinence dans un contexte particulier, en choisissant et en mobilisant un double équipement : ressources personnelles (connaissances, qualités, culture, émotions ...) et ressources de réseau (bases de données, documents, réseaux d'expertise, ...)". Certains auteurs rajoutent à cette définition, la notion de savoir être ou d'aptitudes qui sont d'ordre cognitif et comportemental (Jolis, 1997). Pour (Levy-Leboyer, 1996), "les compétences concernent la mise en œuvre intégrée d'aptitudes, de traits de personnalité et aussi de connaissances acquises pour mener à bien une mission complexe dans le cadre de l'entreprise qui a chargé l'individu, et dans l'esprit de ses stratégies et de sa culture". (Mitrani et al., 1992) étend le savoir être à toutes les caractéristique individuelles et les motivations qui peuvent être mesurées avec fiabilité et qui peuvent se manifester pour différencier de manière significative les sujets dans leurs activités.

La définition souvent admise par les industriels est celle qui a été énoncée par le mouvement des entreprises de France (MEDEF, 2002) comme étant : "une combinaison de connaissances, savoir-faire, expériences et comportements s'exerçant dans un contexte précis. Elle se constate lors de sa mise en œuvre en situation professionnelle à partir de laquelle elle est validable".

Dans la littérature anglo-saxonne, les connaissances et savoir-faire sont qualifiés de "hard competences", les autres catégories relatives aux attitudes, comportements (behaviors), traits de personnalité et motivations sont regroupées sous la catégorie "soft competences". Ainsi par exemple, (Klein, 1996) relie la compétence à un ensemble de comportements observables conduisant à des performances reconnues. 
Cependant une distinction est souvent observée entre les termes "competency" et "skill". Ce dernier correspond à une compétence élémentaire ou une habilité manuelle qui est une composante particulière de la compétence. Dans (Lucia et al., 1999), la compétence est considérée comme une combinaison d'un ensemble de "skills" avec d'autres ressources (connaissances, attitudes, personnalités...). Des définitions similaires sont observées dans (Faucher et al., 2003).

En ce qui nous concerne, nous retenons la définition proposée dans (Bonjour et al., 2002) qui met l'accent sur la finalité, la contextualisation et sur la performance associée à la compétence en terme de valeur pour l'entreprise : "La compétence est la mobilisation d'un ensemble de savoirs hétérogènes aboutissant à la production d'une performance reconnue, par rapport à un environnement donné et dans le cadre d'une activité finalisée".

Cette définition tient compte de trois caractéristiques essentielles de la compétence que nous énonçons ci-dessous :

- La validation d'une compétence suppose la production d'une performance significative, apportant une valeur ajoutée, dans le cadre une activité finalisée (Athey et al., 1999). Cette performance doit être validée par un système de reconnaissance propre à l'organisation (Spencer et al., 1993).

- Toute compétence est sous-tendue par une structure cognitive qui organise la manière dont est conduite l'activité correspondante (Vergnaud, 1998). L'activation d'une compétence fait appel à plusieurs processus cognitifs et manipule des ressources hétérogènes, à la fois cognitives et matérielles.

- La compétence est créée, développée et mise en œuvre à travers des interactions qu'entreprend un acteur avec sa situation. La relation entre les concepts de compétence et de situation de travail est à la base de nos travaux.

Les deux dernières caractéristiques ont été mises en évidence dans des travaux antérieurs de (Bonjour et al., 2002). Ces auteurs présentent un modèle théorique pour l'analyse et la compréhension du fonctionnement d'une compétence. Ce modèle repose sur les apports de la théorie systémique et utilise différentes sources théoriques relatives à la cognition pour l'action, en particulier, la théorie de l'action de (Parson, 1965), la théorie des schémas d'action (Sebillotte, 1998), des théories de la prise de décision (Simon, 1969), (Rasmussen, 1986) et surtout, la théorie de l'activité (Vygtsky, 1978), (Engeström, 1987) et la théorie des schèmes développée par (Piaget, 1975) et reprise par (Vergnaud, 1998). Des auteurs comme (Le Boterf, 2000) et (Vergnaud, 1998) insistent sur la pertinence du concept de schème dans la modélisation des compétences. Piaget définit le schème d'une action comme "la structure générale de cette action, se conservant au cours de ses répétitions, se consolidant par l'exercice, et s'appliquant à des situations qui varient en fonction des modifications du milieu". Par la suite, Vergnaud définit un schème comme l'organisation invariante de la conduite de l'activité, pour une classe de situations. LeBoterf (2002) précise alors que "le schème opératoire, qui constitue la compétence réelle, doit donc se caractériser par une certaine invariance. La stabilité 
du schème est une garantie de compétence". Ces propriétés sont cruciales et constituent les hypothèses de base pour la modélisation d'une compétence.

Dans le modèle proposé par (Bonjour et al., 2002), la relation entre Compétence et \{Situation ; Ressources cognitives ; Organisation de l'action\} est explicitée par différents processus cognitifs supportant la mobilisation de la compétence. Le premier processus est enclenché après la prescription d'une mission. Il concerne l'observation de la situation en cours, l'identification des éléments pertinents dans cette situation par rapport à mission et la recherche des situations antérieures contenant la réalisation d'une mission similaire dans le passé. En fonction du degré de similarité entre la nouvelle situation et les situations passées, l'acteur va pouvoir décider de suivre totalement, ou partiellement, un plan d'actions particulier. Un plan d'actions comporte un ensemble ordonné ou semi-ordonné d'actions. C'est une manière possible d'organiser la réalisation d'une tâche.

Le concept de situation représente un fort intérêt pour nous, du fait que ses éléments sont plus faciles à observer et à représenter que les aspects cognitifs. L'apport des méthodes de traçabilité peut être de grande utilité pour représenter les éléments observables dans une situation de travail. Le paragraphe suivant présente un bref état de l'art relatif à la traçabilité et la gestion des connaissances.

\subsection{Traçabilité et gestion des connaissances}

La gestion des connaissances est un domaine de recherche à part entière (Studer et al., 1998). Il ne s'agit pas d'une simple opération de stockage et d'archivage d'informations mais plutôt d'une capture intelligente des connaissances relatives aux différentes situations de vie de l'entreprise, une structuration de ces connaissances à travers des modèles variés et une meilleure exploitation de toutes ces connaissances par le partage au moment opportun entre les acteurs qui en ont besoin. (Grundstein 2001) a proposé un cycle de gestion de connaissances composé de quatre actions : repérage, préservation, valorisation et actualisation.

En conception, ce cycle se traduit par une double relation entre l'activité des acteurs de conception et les mémoires de connaissances associées (figure 1). Ces mémoires sont alimentées en permanence par les traces de l'activité du concepteur. Une trace de projet fournit l'historique des informations pertinentes du travail effectué et permet de comprendre l'évolution d'un projet ainsi que les décisions prises pendant son déroulement (Matta et al., 1999). Le concepteur à son tour exploite les connaissances capitalisées pour les réutiliser dans des problèmes similaires. Par son activité, le concepteur génère de nouvelles connaissances, qui pourront être traitées pour mettre à jour le contenu de la mémoire. 


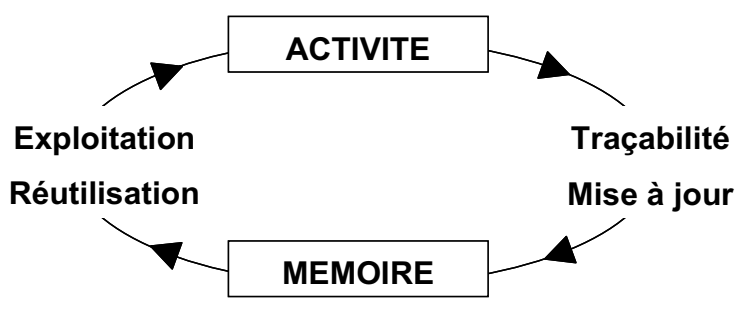

Figure 1. Cycle traçabilité - exploitation en conception

La situation est une source de connaissance, elle comporte un ensemble d'informations ayant un sens pour l'acteur qui va représenter cette situation dans le cadre de son activité (Araujo et al., 2004). Beaucoup de travaux traitent de la modélisation des connaissances relatives à la situation de travail.

Certains modèles ont une finalité d'aide à la résolution de problèmes comme par exemple le "modèle du contexte d'un problème" de la méthode REX (Malvache et al., 1993). D'autres s'intéressent à représenter les différents aspects de la situation collaborative telles que les différents membres du groupe, leur position hiérarchique, les activités qu'ils doivent réaliser, etc. (Borgès et al. 2005).

(Kofod-Petersen et Cassens, 2006) identifient les principales connaissances du contexte de l'activité devant être intégrées dans un système informatique d'aide à la décision. Le modèle de la situation proposé par ces auteurs distingue et regroupe différents types de contexte : le contexte personnel regroupant les différentes informations sur les objets physiques; le contexte social représentant les rôles que doit occuper un acteur; le contexte des tâche définissant les différentes tâches que doit réaliser cet acteur et enfin, le contexte spatio-temporel dans lequel se déroule l'activité. Nous retrouvons dans (Kirsch-Pinheiro et al., 2004) un modèle similaire comportant les éléments à prendre en compte dans la représentation de la situation, tels que les processus, les objets partagés durant la collaboration, les planning, etc.

(Houssin et al., 2006) proposent un modèle de situation permettant de développer une aide à la conception des systèmes de production et à l'évaluation des choix techniques du point de vue de la gestion des risques d'utilisation. Ce modèle conceptuel vise à intégrer, dès la phase de conception, les contextes d'utilisation et le comportement du système de production. Il représente à la fois le système et l'équipe de travail ainsi que d'autres entités (tâches, auxiliaires, zones, risques, etc.) pertinentes pour représenter une activité de conception de systèmes à risques.

D'autres modèles sont présentés dans la littérature pour structurer les connaissances dans une mémoire de projet (Matta et al., 1999). Nous nous inspirons de ces travaux pour construire un modèle, adapté à notre problématique, qui sera présenté dans le paragraphe 5.1 . 


\subsection{Le pilotage des compétences}

Le pilotage des compétences s'intéresse à tous les processus internes qui contribuent à la production, à la mise en œuvre et au développement de compétences spécifiques à une organisation, produisant des biens ou des services.

La structure de pilotage d'un système de compétences d'une entreprise doit porter sur différents horizons temporels et niveaux hiérarchiques (Dulmet et al., 2003). Le pilotage opérationnel nécessite une responsabilisation des acteurs individuels et collectifs pour mobiliser, maintenir et développer leurs compétences. Le pilotage tactique gère la dynamique des compétences en adéquation avec les orientations stratégiques de l'entreprise : définition et mise en place de plan d'actions de développement des compétences, nouvelles structurations des processus ou des acteurs, etc. Enfin, le pilotage stratégique définit les grandes orientations concernant les évolutions majeures en prenant en compte la dynamique possible des compétences et en formulant des besoins en compétences stratégiques de l'organisation. Ce niveau doit être une composante de la stratégie de l'organisation.

Dans le domaine professionnel, le Mouvement des Entreprises de France (MEDEF 2002) a entrepris depuis quelques années une action de sensibilisation et d'accompagnement auprès des industriels afin de les inciter à repenser leurs modes de gestion des ressources humaines et à mettre en place une nouvelle démarche basée sur les compétences. Dans le domaine scientifique, plusieurs axes de pilotage de compétences sont traités pour répondre à ces besoins industriels et économiques. (Stenlund et al., 1999) identifient cinq étapes dans le processus de pilotage des compétences : acquisition, allocation, développement, maintenance et utilisation. En France, les premiers travaux en génie industriel concernent surtout l'intégration du concept de compétence dans la modélisation d'entreprise et des systèmes de conduite (Jia 1998), (Harzallah, 2000), (Boucher et al., 2003), (Houé 2006) ...

Dans (Boucher et al., 2007), nous retrouvons une synthèse sur les différents travaux dans le domaine du pilotage des compétences. Plusieurs axes y sont identifiés. Parmi ces axes, nous retenons les quatre axes les plus importants :

- Identification des compétences disponibles dans une organisation (Harzallah et al., 2006), et valorisation des compétences clés et critiques (core competencies) susceptibles de favoriser l'avantage concurrentiel de cette organisation (Prahalad et al., 1990) afin de garantir leur maintien et leur évolution (Pépiot 2005). A titre d'exemple, (Pépiot, 2005) propose une méthode basée sur la logique floue pour aider à la valorisation des compétences critiques dans une entreprise. Chaque compétence est caractérisée par un ensemble de ressources matérielles et non matérielles de type savoir, savoir-faire et savoir-être. Trois étapes sont préconisées : (1) formuler une grille de critères représentatifs, du point de vue de l'expert, des composants de la compétence. (2) identifier des indicateurs traduisant la contribution de chacun des critères à l'avantage 
concurrentiel, (3) définir la criticité de la compétence par agrégation des différents indicateurs.

- Evaluation des compétences et intégration de ce concept dans la mesure de la performance globale de l'organisation. Cet axe s'intéresse à évaluer le rôle de la ressource humaine et l'impact des pratiques managériales de pilotage des compétences sur l'amélioration de la performance globale (Ahmad et al, 2003), (Boucher et al., 2003), (Jussupova, 2007). (Bennour, 2007) a proposé une méthode pour l'estimation de l'impact de la compétence sur la performance industrielle. La méthode se base sur des lois mathématiques pour intégrer la compétence à travers ses composants : savoir, savoir être et savoir-faire. L'impact de chacune des compétences (élémentaires) nécessaires à la réalisation d'une activité sur la performance nominale est ensuite calculé par une agrégation.

- Allocation des compétences (Canos et al., 2004), et team building (Gronau et al., 2007) (Tseng, 2004), (Hadj-Hamou, 2004). Cet axe concerne l'allocation des ressources humaines aux missions qui correspondent le mieux à leurs compétences et la construction d'équipes de travail performantes. (De Korvin et al., 2002) proposent une méthode pour la définition des équipes de projet sur la base des compétences individuelles des acteurs et leur compatibilité (ou capacité) à travailler ensemble dans un projet. L'approche utilise le principe de compatibilités floues et se déroule selon les grandes étapes suivantes (1) décrire les phases du projet en terme de compétences requises et les acteurs candidats en termes de compétences acquises, (2) calculer les différents indices de compatibilité pour chaque couple de compétences acquise/requise, (3) sélectionner la composition qui optimise les indices.

- La mise en œuvre et le développement des compétences, qui s'intéressent au mode de transfert des compétences et aux conditions d'émergence d'une compétence collective. Cette thématique est surtout traitée par des approches socio-cognitives (Oget et al., 2001), (Vaudelin, 2005) et par des travaux sur l'impact de la reconnaissance des compétences sur les conditions sociales du travail (Pierre et al., 2007), (Cavestro et al., 2007).

\subsection{La caractérisation des compétences en vue du pilotage}

Les démarches de pilotage des compétences utilisent souvent une étape préliminaire de caractérisation de la compétence à travers des composants évaluables par des mesures quantitatives ou qualitatives indiquant le niveau de maitrise de ces composants. Cette étape permet de manipuler le concept de compétence qui est de nature abstraite par des éléments tangibles pouvant être représentés et évalués.

Ainsi par exemple, nous retrouvons dans certaines approches de valorisation des compétences, une caractérisation de la compétence par ses différents composants puis une caractérisation de ces composants par leur degré de criticité par rapport aux 
objectifs de l'entreprise, ceci pour évaluer un niveau global de compétence (Boucher et al., 2003) ou la valeur de la criticité de la compétence par rapport aux objectifs concurrentiels (Pépiot, 2005). Les approches d'allocation cherchent à minimiser les distances entre les caractéristiques des compétences acquises par ces acteurs et celles des compétences requises par les missions (Fitzpatrick et al., 2005), (De Korvin et al., 2002). L'étape de caractérisation est également réalisée en amont, lors de la définition des actions de développement de compétences, pour identifier les aspects à faire évoluer pour atteindre un certain niveau de maîtrise d'une compétence.

La caractérisation des compétences consiste plus précisément à :

- Définir une typologie de composants de compétences,

- Définir une échelle d'évaluation des niveaux de maîtrise,

- Développer une méthode et une procédure pour la caractérisation.

La définition d'une typologie de composants d'une compétence dépend de la spécificité du domaine d'activité. Plusieurs typologies ont été proposées dans la littérature pour désigner les composants d'une compétence (Acuna et al., 2004), (Ley, 2006), ... La typologie la plus utilisée en GRH et en Génie Industriel est le triplet : savoir, savoir-faire et savoir-être.

Pour l'évaluation des niveaux de maîtrise des compétences, plusieurs échelles de mesure sont proposées dans la littérature comme celle de (Michel et al., 1991) qui comporte trois niveaux de capacités cognitives : application ("je sais appliquer"), adaptation ("je sais adapter") et création ("je sais innover"). (Pépiot 2005) a proposé une typologie avec quatre niveaux pour décrire la maîtrise du savoir : notion, aisance, expertise, formation. D'autres auteurs se contentent d'évaluer le niveau de compétence par des niveaux symboliques (faible, moyen, etc.) ou numériques (note).

La dernière catégorie de contributions à la caractérisation concerne le développement de nouvelles démarches pour aider les experts "managers" dans leurs missions de définition des référentiels de compétences.

La caractérisation des compétences est intrinsèquement liée à la problématique de construction des référentiels de compétences. Le référentiel de compétences permet à l'entreprise de structurer et de stocker des informations sur, d'une part, les compétences acquises par les employés et d'autre part, les compétences requises pour réaliser les missions qui constituent le cœur de métier de l'entreprise.

\subsection{Méthodes empiriques pour la construction des référentiels de compétences}

Les méthodes de caractérisation et de construction des référentiels de compétences émanent principalement du domaine du management des ressources humaines. Nous retrouvons dans (Rault, 1993) une revue des principales méthodes françaises telles que la démarche fonction, développée par l'A.P.E.C (1979), la démarche "Développement et Emploi" (1981), la démarche cognitive, développée 
par C.O.R.O.M. (1987), la démarche ergonomique, développée par l'A.N.A.C.T. (1990) et la démarche formation, du C.N.A.M. (1991).

La majorité de ces méthodes repose sur une étape fondamentale d'analyse des situations de travail dans lesquelles la compétence va être mobilisée. Nous retenons par exemple, le guide méthodologique pour l'élaboration des référentiels d'"emploi - activités - compétences", mis en œuvre par la direction du développement des compétences du centre national de la fonction publique territoriale (CNFPT, 2001). Après analyse des autres approches, nous avons retenu cette démarche qui présente l'avantage d'être synthétique et représentative du processus générique de caractérisation. Sa simplicité la rend opérationnelle et bien adaptée à "une automatisation". Elle se décompose en quatre étapes :

- Étape 1 : Recueil de données sur les emplois et les activités et son environnement (situation de travail).

- Étape 2 : Analyse des données précédentes, afin d'en tirer les indicateurs communs et d'en dégager les spécificités.

- Étape 3 : Déduction des compétences par la définition de la combinaison savoirs, savoir-faire et qualités nécessaires à la réalisation de chaque type d'activité selon les spécificités identifiées.

- Étape 4 : Traitement et capitalisation des données dans des référentiels pour de futures utilisations en gestion des compétences.

La majorité des méthodes utilise des outils informels qui réduisent l'objectivité du processus de caractérisation et de construction des référentiels. L'approche de caractérisation des compétences que nous proposons sera structurée à partir de ces quatre étapes. Chaque étape sera supportée par un module de traitement de données permettant d'automatiser le processus de caractérisation. Ceci permettra de donner plus de fiabilité dans la représentation et la manipulation des données de la situation.

\section{Démarche de caractérisation des compétences}

Dans cette section, nous présentons une démarche globale de caractérisation. Pour cela, nous revenons d'abord sur le support théorique et sur l'hypothèse de base qui nous a permis de prendre en compte le concept de situation dans la démarche de caractérisation. Sur la base de cette hypothèse, nous présentons les modules essentiels du système de caractérisation. Ces modules seront ensuite explicités progressivement dans les sections suivantes.

Il est à noter que le principe de solution proposé est générique. Cependant, le cas d'application de la démarche concerne le domaine de la conception. Les éléments identifiés et manipulés dans la description de la démarche sont des caractéristiques d'une situation de conception ( $C f$. partie 5.2) e t ont été jugés pertinents, dans cette application, pour décrire la compétence d'un concepteur. 


\subsection{Hypothèse de base de la méthode}

L'importance du concept de situation de travail est une conséquence de la forte relation qui existe entre les concepts de schème, de compétence et de situation de travail $(C f . \S 2.1 .2)$. Les interactions entre l'acteur au cours de son activité et la situation sont la base du développement et du fonctionnement du schème qui supporte la compétence. L'acquisition de la compétence nécessaire pour la réalisation de l'activité revient dans ce sens à la maîtrise de tous les aspects relatifs aux différentes interactions produites durant la réalisation de l'activité.

Après la prise en charge de sa mission, l'acteur est en interaction avec différents éléments de sa situation à travers des entités interactionnelles (notée E.I.j dans la figure 2) pour bien réaliser son activité. La réussite de l'activité permet d'induire de la part de l'acteur, d'un côté, un certain niveau de maîtrise des ressources matérielles mises à sa disposition, et d'un autre côté, un potentiel cognitif et un comportement relationnel satisfaisants.

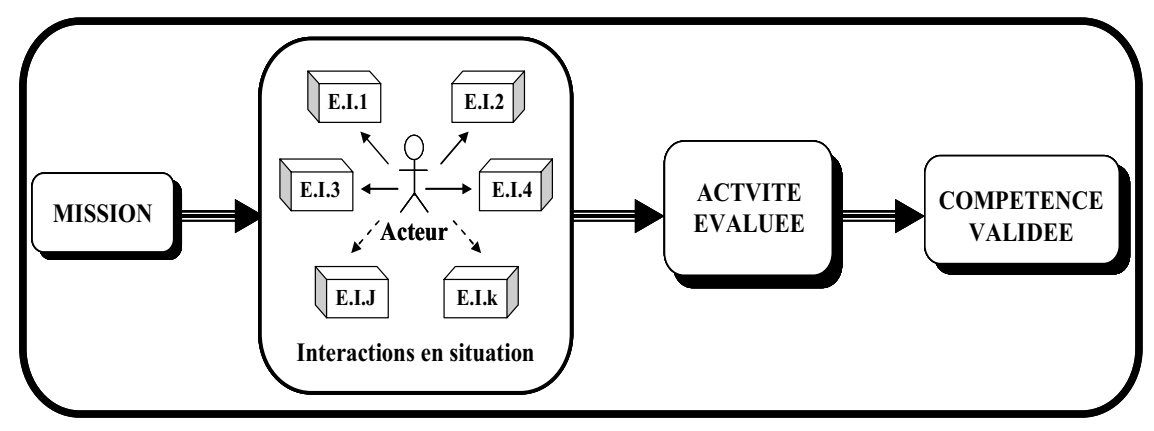

Figure 2. Hypothèse de base de la méthode

Nous supposons donc que le niveau d'une compétence validée doit être mesuré en fonction de ces différentes interactions. Ce qui revient à supposer que : "pour aboutir aux résultats escomptés de l'activité, il a suffi de mettre en œuvre les niveaux "Ni" des composants de compétence "Ci" en correspondance avec la nature des interactions produites durant l'activité". Dans ce cas, après évaluation de l'activité, celle-ci est validée si ses résultats sont jugés satisfaisants. L'hypothèse de base de notre approche est la suivante : A chaque composition de situation (entitésinteractions) correspond au moins une combinaison de caractéristiques de cette situation. Ces caractéristiques sont relativement stables dans une classe de situations (Vergnaud, 1998) et permettent d'avoir une relation de la forme :

Éléments de la Situation $\Rightarrow$ composants de la Compétence. 


\subsection{Les étapes de la démarche}

Nous proposons de caractériser une compétence validée à partir des traces d'une activité réellement effectuée dans une situation de travail (Belkadi et al., 2007). La méthode s'articule en trois étapes complémentaires, comme le montre la figure 3 :

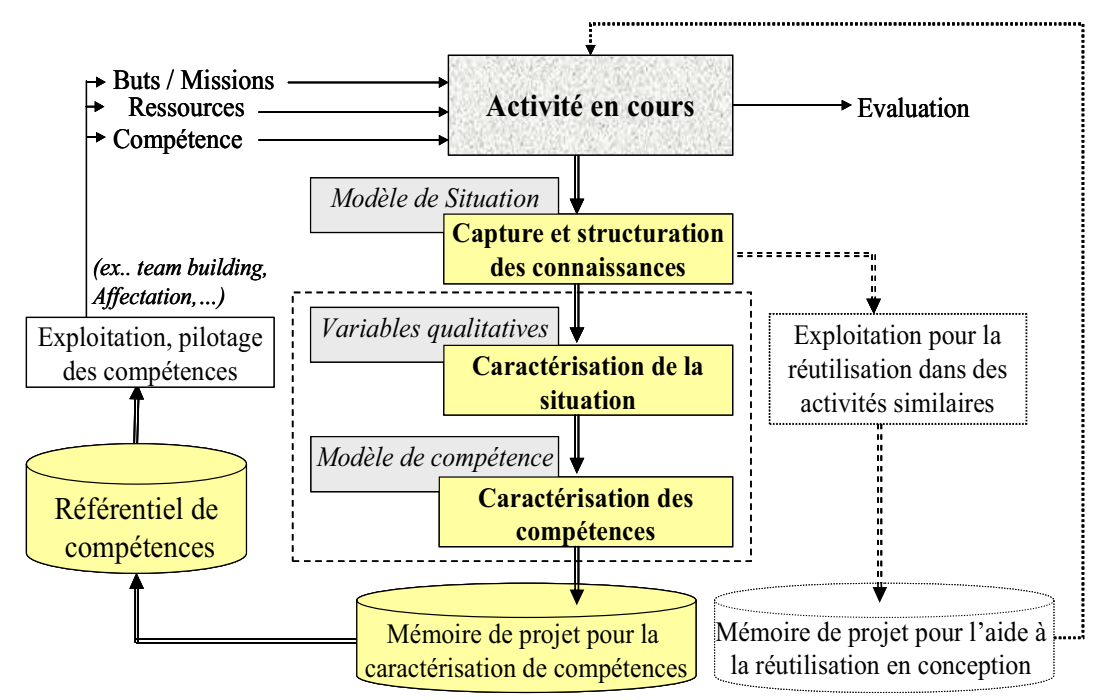

Figure 3. Principe général de l'approche de caractérisation des compétences

- La première étape "Capture et structuration des connaissances" comporte la spécification d'un système de traçabilité pour la capture et la structuration des connaissances sur la situation de travail au cours d'un projet de conception. Cette spécification est faite par une modélisation UML de tous les aspects présents (ou susceptibles de l'être) dans une situation. Il s'agit des aspects relatifs à la définition du produit, à l'organisation de l'activité, aux échanges coopératifs, aux décisions, etc. De ce fait, une autre sortie peut être envisagée en parallèle pour capitaliser les connaissances à des fins de réutilisation et de résolution de problèmes.

- La deuxième étape "Caractérisation de la situation" constitue l'étape de prétraitement dans le processus de caractérisation des compétences. Il concerne la caractérisation de la situation via des variables qualitatives faciles à manipuler dans l'optique de caractérisation des compétences.

- La dernière étape "Caractérisation des compétences", concerne le processus de caractérisation des compétences à partir de l'analyse de la situation de travail. La sortie du module est une évaluation des composants de la compétence, 
définis selon un modèle théorique de la compétence. Pour ce module, nous proposons d'utiliser un traitement flou.

L'étape de caractérisation des compétences est associée à un module de traitement flou et à un module de sortie fournissant et structurant les résultats de caractérisation (caractéristiques des composants de la compétence).

La logique floue est souvent utilisée dans les systèmes d'aide à la décision qui manipulent des données qualitatives dans des contextes entachés d'imprécision et d'incertitude (Bouchon-Meunier et al., 2003). L'intérêt d'utiliser un tel formalisme dans notre démarche est double :

- L'usage des variables de type linguistique est nécessaire pour représenter le jugement qualitatif de l'expert et pour caractériser la situation. La valeur d'une variable linguistique traduit un aspect imprécis (Herrera \&al., 1994), (Lesage 1997) (par exemple, une tâche peut être jugée : "simple", "relativement complexe" ou "difficile").

- Les règles d'inférences manipulent des variables linguistiques pour représenter un raisonnement particulier proche de celui d'un expert (par exemple, un raisonnement qualitatif d'un manager peut être du type : si une tâche est "difficile", alors prévoir des ressources "importantes").

Dans notre approche, le module de traitement flou permet de faire le lien, à travers des règles d'inférences, entre les caractéristiques de la situation et celles des compétences associées.

La figure 4 représente la structure générale de ce module. Les étapes de capture des connaissances et de caractérisation de la situation constituent le module d'entrée en amont du module de traitement flou qui comporte les trois phases de fuzzification, d'inférences et de défuzzification.

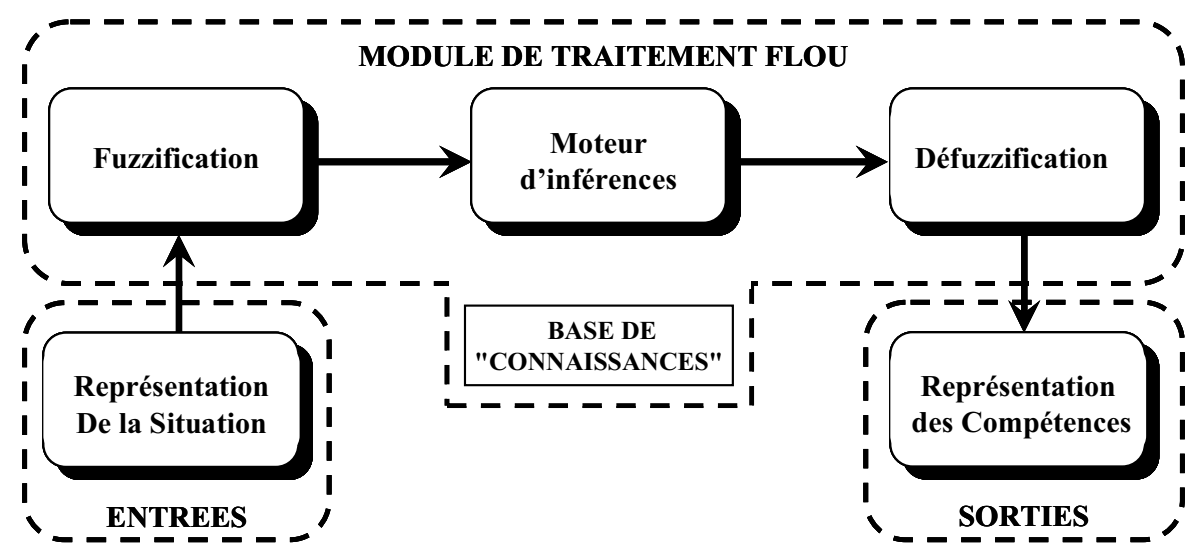

Figure 4. les trois étapes du traitement flou de caractérisation des compétences 
Le tableau 1 regroupe les liens entre les différentes étapes proposées dans le système de caractérisation et les étapes rencontrées dans les approches empiriques.

\begin{tabular}{|c|l|l|}
\hline Module & \multicolumn{1}{|c|}{ Etape automatique } & \multicolumn{1}{c|}{ Etape empirique } \\
\hline \multirow{2}{*}{$\begin{array}{c}\text { Entrée : } \\
\text { représentation de } \\
\text { la situation }\end{array}$} & $\begin{array}{l}\text { Capture et structuration des } \\
\text { connaissances de la situation }\end{array}$ & $\begin{array}{l}\text { E1 : Recueil de données } \\
\text { sur l'activité et sa situation. }\end{array}$ \\
\cline { 2 - 3 } & Caractérisation de la situation & $\begin{array}{l}\text { E2 : Analyse qualitative } \\
\text { des données sur la situation }\end{array}$ \\
\hline \multirow{2}{*}{ Traitement flou } & Fuzzification & E3 : Déduction des \\
\cline { 2 - 2 } & Inférences & Dempétences \\
\cline { 2 - 3 } $\begin{array}{c}\text { Sortie : } \\
\text { représentation de } \\
\text { la compétence }\end{array}$ & $\begin{array}{c}\text { Caractéristiques des } \\
\text { composants de la compétence }\end{array}$ & $\begin{array}{l}\text { E4 : Structure du } \\
\text { référentiel de compétence. }\end{array}$ \\
\hline
\end{tabular}

Tableau 1. Relations étapes automatiques et étapes empiriques de caractérisation

Dans les sections suivantes, nous présentons les étapes de notre approche. Nous détaillons ensuite un exemple illustratif du processus de caractérisation de la compétence associée à une activité technique dans un processus de conception.

\section{Module de sortie : Représentation de la compétence}

Le module de sortie décrit le résultat du système de caractérisation d'une compétence. Sa structuration guide, par la suite, le choix des variables d'entrée ainsi que le choix des règles d'inférence. Nous commençons donc par sa description.

L'analyse de l'activité des concepteurs et la représentation du fonctionnement d'un schème (Bonjour et al., 2002) permettent de mettre en évidence deux types de composants importants : les connaissances techniques et les capacités opératoires ou relatives aux règles de conduite, qui se décrivent ainsi :

- Les connaissances techniques :

- "Opérationnelles", relatives aux besoins d'interprétation et de mise en œuvre : contraintes de réalisation de la mission, procédures de réalisation ...

- "Support", relatives aux besoins de manipulation des entités servant de support lors de l'interaction, par exemple : les référentiels méthodologiques, le mode d'emploi des outils, l'outil CAO ... 
- "Objet", nécessaires à la manipulation de l'objet traité par l'interaction.

- Les capacités opératoires :

- "d'Analyse", qui permettent d'appréhender la situation et de pouvoir en tirer les informations pertinentes pour réaliser l'activité : identifier les relations, faire des synthèses ...

- "d'Organisation", qui permettent de définir ou modifier le plan d'actions et d'organiser les ressources de l'activité : planifier, décomposer ...

- Les capacités relatives aux règles de conduite :

- "Relationnelles", nécessaires pour mener des activités dans un contexte collaboratif: respecter les règles d'une communauté, exprimer ses idées, argumenter et justifier ...

- "Décisionnelles", permettant d'identifier les critères de décision, de les classifier, de choisir, de réagir aux aléas ...

Chacun de ces composants de la compétence est évalué par une mesure qualitative relative traduisant une estimation du niveau de maîtrise de la compétence par rapport à ce composant en fonction de ce qui est observé dans la situation correspondante. L'objectif de notre démarche est d'inférer le résultat de ces mesures à partir d'une évaluation des caractéristiques de la situation réelle.

\section{Le module d'entrée : représentation de la situation}

Dans ce premier module, les données issues de la situation sont structurées selon des modèles permettant de représenter les connaissances utiles au processus de caractérisation des compétences. L'analyse de ces données est obtenue par une qualification des différentes caractéristiques de la situation.

\subsection{Modèle de la situation}

Dans la littérature, plusieurs formulations ont été utilisées pour définir le concept de situation (DeTerssac 1998), (Livian 1998), (CNFPT 2001). Nous retrouvons dans ce concept les caractéristiques clés définissant un système au sens de Le Moigne (1990) et de Searle (1998) que nous résumons ici. (1) Au regard d'un observateur ou d'un acteur qui définit sa situation, celle-ci est identifiée comme quelque chose qui a un sens et une cohérence justifiée par les liens de causalité entre les différents éléments de la situation. (2) Ces éléments et liens de causalité forment la structure $\mathrm{du}$ "système situation". Cette structure sera délimitée par le champ perceptif ou de focalisation de l'acteur ou de l'observateur qui représente cette situation. (3) La principale fonction du "système situation" est de fournir une aide à l'acteur pour (se) représenter le monde qui l'entoure et comprendre les liens qu'il a avec ce monde. (4) Enfin, cette représentation de la situation n'a de raison d'être, aux yeux d'un acteur, que par rapport à une finalité d'action de cet acteur sur le "système situation". 
Nous nous sommes basés sur ces caractéristiques pour donner une définition de la situation, également conforme à l'hypothèse de base de notre méthode $(C f$. $\$ 3.1)$. Ainsi, l'approche systémique nous permet de considérer la situation comme un espace d'interactions entre des éléments de différentes natures : matériels (objets physiques, outils, ...) ou cognitifs (individus, modèles, ...). Nous proposons de décrire la situation de déroulement d'une activité par un ensemble d'entités participant par des rôles spécifiques à une ou plusieurs entités interactionnelles (Belkadi et al., 2004).

Le modèle générique de la situation (figure 5) reflète cette représentation. La relation d'héritage permet d'intégrer une typologie de cinq rôles spécifiques. Il s'agit des rôles acteur, client, manager, support et objet.

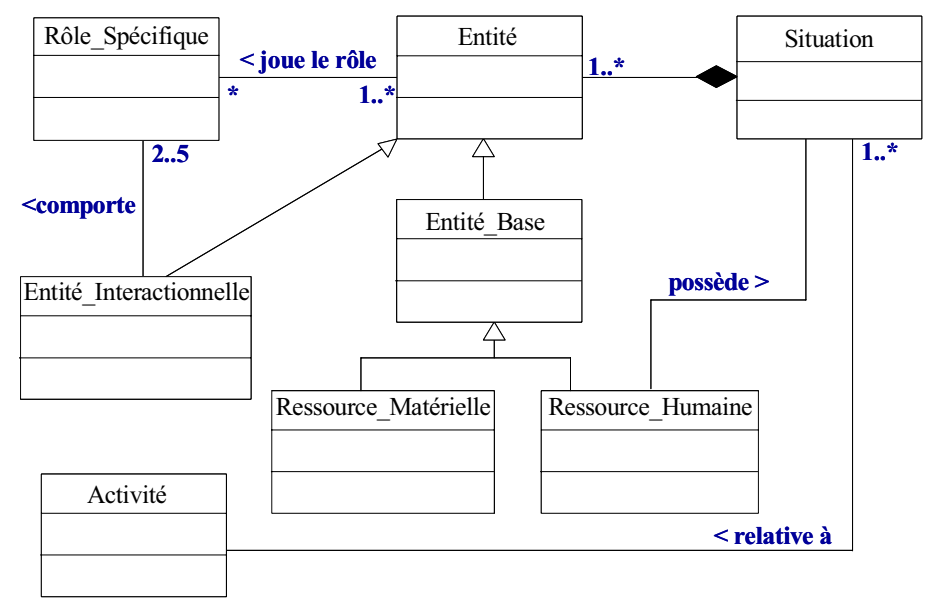

Figure 5. Modèle générique de la situation

Nous avons distingué deux types d'entités :

- Les entités de base : (notées "EB") représentent les aspects physiques de la situation tels que le produit, les différents acteurs humains présents dans l'environnement ou encore les différentes ressources matérielles ...

- Les entités interactionnelles: (notées "EI") représentent les différents liens entre les entités de la situation. Ces entités sont classées en quatre catégories complémentaires :

- Les "E.I" communautaires représentent la relation d'appartenance des entités humaines à une certaine communauté (Exemple : équipe projet, département).

- Les "E.I" transactionnelles décrivent les mécanismes d'échange d'informations entre les acteurs (Exemple : communication, coopération). 
- Les "E.I" opérationnelles servent à la fois à la définition des prescriptions et à la description de la réalisation de ces prescriptions (Exemple : tâche, mission, processus, activité).

- Les contraintes permettent de formuler une limitation sur la réalisation d'une interaction donnée (Exemple : contraintes organisationnelles, règles de conception).

Ce modèle est ensuite décliné en différents sous modèles correspondant à différents types d'interactions qui interviennent dans la définition des composants d'une compétence (Belkadi 2006) :

- Les différentes entités de base présentes dans la situation,

- L'organisation des entités humaines dans les structures organisationnelles,

- L'organisation du projet à travers ses différents objectifs,

- Les activités réalisées par les acteurs individuels ou collectifs pour atteindre ces objectifs ainsi que la manière par laquelle ont été réalisées ces activités (actions élémentaires et plans d'actions),

- Les transactions collaboratives menées par les acteurs durant leurs activités,

- Les résultats réellement produits par les activités (modification des entités)

La classe "Rôle_Spécifique" est une classe générique représentant grâce à des relations d'héritage, la contribution de chaque entité aux différentes interactions. Cinq types de rôles sont définis à savoir :

- Le rôle "Acteur" qui est attribué à l'entité responsable du résultat de l'interaction (réalise une activité par exemple),

- Le rôle "Client" qui concerne l'entité qui spécifie le besoin de l'interaction et reçoit son résultat,

- Le rôle "Manager" qui est attribué à l'entité qui régule le fonctionnement d'une interaction,

- Le rôle "Support" qui est attribué à l'entité qui fournit une aide dans l'interaction,

- Le rôle "Objet" qui concerne l'entité directement concernée par le résultat d'une interaction.

\subsection{Qualification des caractéristiques de la situation}

Le module de caractérisation de la situation est une étape de prétraitement visant à générer de nouvelles connaissances ayant "un sens" par rapport au besoin de caractérisation des compétences. Ces connaissances seront représentées à l'aide de variables spécifiques traduisant l'appréciation d'un expert.

Cependant, le jugement de l'expert est généralement exprimé en langage naturel. L'usage des variables de type linguistique (Zadeh 1965), (Dubois et al. 1997), 
(Zimmermann, 2001) (Bouchon-Meunier et al., 2003) est nécessaire pour représenter l'aspect qualitatif du jugement.

\begin{tabular}{|c|c|c|c|c|c|}
\hline \multirow{2}{*}{\multicolumn{2}{|c|}{$\begin{array}{c}\text { Composants } \\
\text { de la Compétence }\end{array}$}} & \multicolumn{4}{|c|}{ Caractéristiques de la situation } \\
\hline & & $\begin{array}{l}\text { Complexité } \\
\text { (Compl.) }\end{array}$ & $\begin{array}{l}\text { Nature de } \\
\text { l'activité }\end{array}$ & $\begin{array}{c}\text { Nature } \\
\text { relationnelle }\end{array}$ & $\begin{array}{l}\text { Entités } \\
\text { et rôles }\end{array}$ \\
\hline \multicolumn{2}{|c|}{$\begin{array}{l}\text { Connaissances } \\
\text { techniques }\end{array}$} & & $\begin{array}{l}\text { Proximité } \\
\text { / Exécution }\end{array}$ & $\begin{array}{c}\text { Fréquence } \\
\text { d'interactions }\end{array}$ & $\begin{array}{c}\text { Rôles et } \\
\text { ressources }\end{array}$ \\
\hline \multirow{7}{*}{ 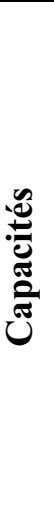 } & Analyse & Compl. / objet & $\begin{array}{l}\text { Proximité } \\
\text { / analyse }\end{array}$ & & $\begin{array}{l}\text { Aspects } \\
\text { produit }\end{array}$ \\
\hline & \multirow{2}{*}{ Organisation } & Compl. / actions & & & Rôles \\
\hline & & Compl. / entités & & & Entités \\
\hline & \multirow[b]{2}{*}{ Décision } & \multirow{2}{*}{$\begin{array}{l}\text { Compl. / } \\
\text { contraintes }\end{array}$} & \multirow{2}{*}{$\begin{array}{l}\text { Proximité } \\
\text { / Décision }\end{array}$} & & Contraintes \\
\hline & & & & & $\begin{array}{c}\text { Plan } \\
\text { d'actions }\end{array}$ \\
\hline & \multirow{2}{*}{ Relation } & & & Type_transaction & Rôles \\
\hline & & & & Nbre_participants & Ress. Hum. \\
\hline
\end{tabular}

Tableau 2. Caractéristiques de la situation et composants de la compétence

L'objectif de notre approche est de s'appuyer sur les données fournies par un système de traçabilité pour caractériser la situation globale dans laquelle s'est déroulée l'activité. L'estimation des valeurs linguistiques est obtenue par une instanciation calculée à partir des données tracées dans la situation. Nous proposons une sélection de variables linguistiques pour caractériser la situation. Nous définissons chaque variable par une expression mathématique de ces données.

Le tableau 2 résume les différents liens que nous avons identifiés entre les caractéristiques de la situation et les composants de la compétence. Dix variables linguistiques ont été ainsi définies représentant la situation à travers sa complexité, la nature de l'activité et la nature relationnelle des transactions relatives à cette activité. Chacune de ces variables participe à son tour à l'estimation du niveau de maîtrise d'un composant de la compétence associée à cette activité.

Nous ne pouvons pas présenter dans cet article l'ensemble des variables. Aussi nous limitons nous à la présentation des variables relatives à la "nature de l'activité" et de la variable "Type_transaction", pour montrer la logique de leur construction. 


\subsubsection{Variables relatives à la "nature de l'activité"}

Certains composants de la compétence renvoient à la nature de l'activité. L'activité peut être modélisée par un plan d'actions qui combine un ensemble d'actions élémentaires, représentant la manière par laquelle l'acteur prévoit et effectue la réalisation de cette activité. Pour cela, nous considérons que toute activité correspond à une résolution de problèmes faisant appel à des actions élémentaires qui se répètent sur des objets différents.

Cinq formes d'actions élémentaires ont été retenues dans notre approche en cohérence avec la théorie de la décision en conception ou de l'artificialisme (Simon, 1969), avec des modèles théoriques de la conception (Pahl et al., 1996) et avec le concept de tâches génériques de conception (Chandrasekaran et al., 1990) :

- Analyse / Compréhension : Le but de cette action est d'extraire de nouvelles connaissances de l'objet de l'étude (ou du problème à résoudre).

- Recherche / Génération (ou proposition) : Cette action a pour résultat la génération et la proposition de nouvelles idées, de nouvelles hypothèses, de nouveaux principes de solution, ...

- Mesure / Evaluation : Cette action permet d'obtenir de nouvelles informations sur les propriétés de certaines entités et de les classifier par rapport à certains critères, pour aider l'acteur dans sa prise de décisions.

- Choix / Décision : L'action de décision peut avoir un effet (direct ou indirect) sur au moins un état d'une des entités de la situation. Elle traduit une alternative choisie par l'acteur pour résoudre son problème.

- Traitement / Exécution : Cette action produit des effets immédiats sur la situation. Il s'agit de transformations, physiques ou informationnelles, sur l'objet de l'activité, qui sont observables par d'autres acteurs de la situation.

La nature de l'activité est définie par trois variables de type "proximité/ X " qui reflètent le type d'actions élémentaires réellement effectuées dans le plan d'actions de l'activité. Nous avons retenu l'aspect X de type "analyse", "décision" ou "exécution". Ces variables sont calculées par l'expression générique suivante :

$$
\text { prox }_{-} X=\frac{\sum_{i=1.5}(\text { nombre d'actions de type } i) \times P_{i}}{\text { nombre total d'actions }}
$$

Avec $\mathrm{i}$ variant de 1 à 5 et $\mathrm{P}_{\mathrm{i}} \in[0,1]$ étant une pondération qui représente la distance qualitative de l'action i par rapport à l'aspect $\mathrm{X}$.

Considérons par exemple la variable "Proximité/ Analyse". 
Une activité comportant une forte concentration d'actions de type "analyse" aura une forte valeur pour la variable "proximité par rapport à l'aspect Analyse". Cette activité est relativement complexe du point de vue cognitif et nécessite une forte capacité d'analyse. On associe donc à ces actions une pondération maximale $(=1)$.

Les actions de type Recherche/génération de solutions sont aussi des actions proches de l'aspect analyse. On leur associe une pondération importante $(=0,9)$. La forme finale de l'expression 1 instanciée sur l'aspect analyse sera la suivante :

\section{Prox_Analyse $=(1 x$ (nbre d'actions analyse $)+0.90 x$ (nbre d'actions recherche $)$ \\ + 0.60x(nbre d'actions décision) + 0.50x(nbre d'actions évaluation) + $0.15 x$ (nbre d'actions traitement)) /(nombre total d'actions)}

\subsubsection{Variable "Complexité / l'objet"}

La complexité par rapport à l'objet se définit selon les vues du produit manipulées durant l'activité : fonctionnelle, comportementale, structurelle et paramétrique. Nous distinguons quatre niveaux de complexité associés à des pondérations entre 0 et 1 . Ces niveaux représentent le degré d'abstraction nécessaire pour traiter les différentes vues du produit. Nous faisons l'hypothèse que les activités qui portent sur un point de vue conceptuel ou fonctionnel nécessitent plus d'abstraction de la part de l'acteur et donc sont plus complexes à analyser que celles portant sur le point de vue comportemental, structurel puis finalement paramétrique. Cette hypothèse se vérifie dans les bureaux d'études puisque la pratique de l'analyse fonctionnelle reste difficile, les concepteurs ayant tendance à penser rapidement en termes de solution technique. Un objet traité uniquement selon le point de vue paramétrique est jugée relativement "simple" par rapport au niveau d'abstraction (cependant, il n'est pas forcément simple à traiter). Nous associons, à chaque point de vue, un facteur de pondération de la façon suivante :

$$
(1 \text { pour Conceptuel }=\text { Fonctionnel })>(0.70 \text { pour Comportemental })>
$$$$
(0.60 \text { pour Structurel })>(0.20 \text { pour Paramétrique })
$$

L'expression de la complexité de l'objet se calcule par la formule suivante :

$$
\text { Compl_ }_{-}=\frac{\sum_{i=1.4}\left(\text { nbre d'objets / po int de vue concerné } e_{i}\right) \times P_{i}}{\text { nombre total d'objet }}
$$


avec $\mathrm{i}$ variant de 1 à 4 et $\mathrm{P}_{\mathrm{i}} \in[0,1]$ étant une pondération qui représente la distance qualitative de l'objet par rapport au point de vue concerné.

Ou plus explicitement :

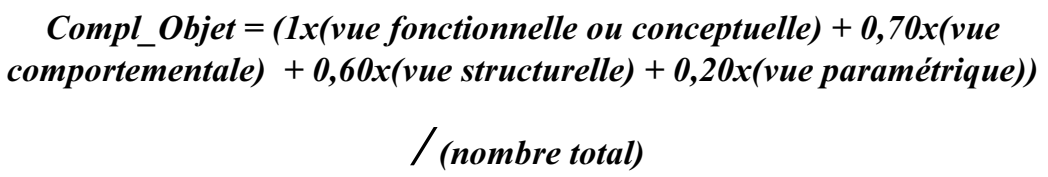

\subsubsection{Variable "Type_transaction"}

Par la même logique, nous construisons la variable descriptive du type de transaction. Plusieurs formes collaboratives sont observées durant un processus de conception. Nous avons retenu les trois formes suivantes : communication, coopération par échange d'information et négociation. Le besoin d'une capacité relationnelle est le plus important pour la dernière forme. Nous avons opté pour l'expression suivante de la variable "Type_Transaction" :

\footnotetext{
Type_Transaction $=(1 \mathrm{x}($ nbre de coopérations par négociation $)+$ $0.50 \times($ nbre de coopérations par échange $)+0.10 \times($ nbre communications) $)$ / (nombre total de transactions durant l'activité)
}

\subsubsection{Variable "Nbre_Participants"}

Le nombre de participants est utilisé pour la définition de la complexité de la transaction. Le poids associé à ce nombre est calculé en fonction du rapport hiérarchique de chacun des participants avec l'acteur de l'activité. Nous faisons ici l'hypothèse que la capacité relationnelle doit être plus importante quand le rôle hiérarchique de l'acteur de l'activité est supérieur à celui des autres participants (statut I, hiérarchie descendante, par exemple : manager vs acteur ou support). Elle est relativement moins importante dans le cas inverse (statut II, hiérarchie ascendante, par exemple : acteur vs manager). Et enfin moins exigeante dans le cas où les rôles hiérarchiques sont égaux (statut III, sans hiérarchie comme par exemple acteur - acteur). La valeur de cette variable est obtenue par l'expression suivante : 
Nbre_Particpant $=(1 \times($ nbre participants statut I $)+0.70 \times($ nbre participants statut II $)$

$+0.20 \times($ nbre participants au statut III))

/(nombre total de participants)

Le même raisonnement a été respecté pour la définition des autres expressions mathématiques. Ces variables sont ensuite instanciées comme des valeurs d'entrée du module de traitement flou que nous présentons ci-après.

\section{Le module de traitement flou}

L'utilisation des principes de la logique floue permet de modéliser le raisonnement subjectif de l'expert "manager" qui traite des informations qualitatives pour la caractérisation des compétences et l'élaboration des référentiels.

La structure du module de traitement flou que nous avons choisie est celle d'un système automatique à base de règles floues de type Mamdani (Dronkov et al., 1993). Les opérateurs logiques utilisés dans ce système pour calculer les résultats des inférences nous semblent appropriés car notre approche de caractérisation consiste à trouver la valeur minimale de la compétence garantissant le résultat validé et décrit par la situation.

La structure générale comporte trois phases de traitement : fuzzification, moteur d'inférences et défuzzification. Une base de connaissances est définie en association avec chaque phase pour décrire les fonctions d'appartenance et les règles d'inférence du système.

\subsection{Fuzzification}

Dans la base de connaissances, il est nécessaire de définir les formes des partitions floues des variables d'entrée et des variables de sortie. Chaque variable de sortie représente les niveaux de maîtrise d'un composant de la compétence (on parlera de la signature de la compétence).

La première étape du traitement consiste à associer à chacune des valeurs réelles en entrées (obtenues à partir de l'instanciation des variables précédentes), le degré d'appartenance par rapport aux valeurs floues correspondantes.

Pour les variables caractéristiques de la situation, nous avons choisi des fonctions d'appartenance d'une forme trapézoïdale. Les valeurs réelles sur l'axe des abscisses ont été normalisées sur une échelle de 0 à $100 \%$. Sur l'axe des ordonnées, nous retrouvons les valeurs d'appartenance $\mu_{\mathrm{A}}(\mathrm{x})$ variant sur $[0,1]$ où $\mathrm{A}$ correspond à la 
valeur floue de la variable et $\mathrm{x}$ à la valeur réelle de la variable, calculée à partir de l'une des précédentes équations. La figure 6 montre l'exemple de la fonction d'appartenance des variables de la catégorie proximité.

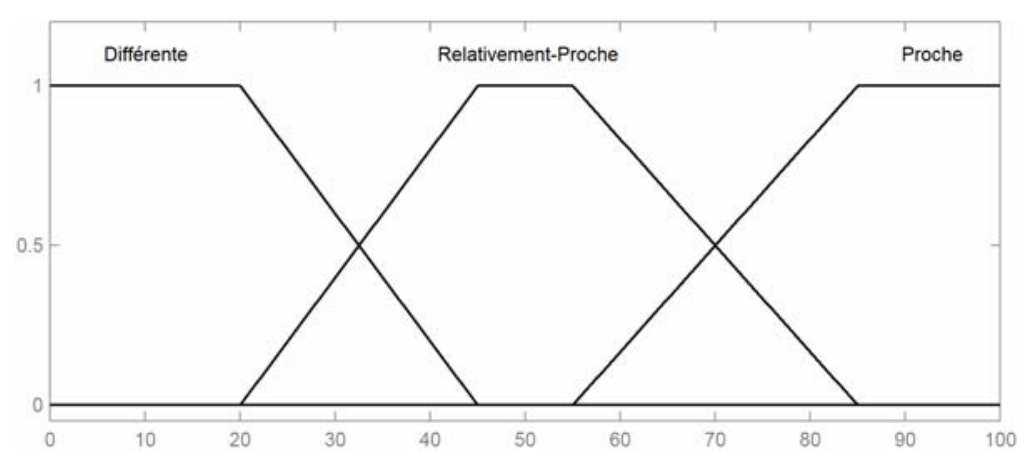

Figure 6. Fonctions d'appartenance pour les variables "proximité"

\subsection{Moteur d'inférences}

L'étape d'inférences consiste à faire les correspondances (Entrées fuzzifiées Sorties fuzzifiées) à partir des règles d'inférences de type IF-THEN. Ces règles ont pour but de représenter le raisonnement subjectif de l'expert manager chargé de la caractérisation des compétences et de la définition des référentiels associés.

Dans la base de "connaissances" associée au moteur d'inférences, nous définissons les différentes règles d'inférences pour chaque combinaison "caractéristiques de situation - composant de compétence" (voir tableau 2). Nous avons défini toutes les règles sous la forme :

\section{Si caractéristique situation 1 ET caractéristique situation 2 ALORS \\ Niveau du composant de compétence associée est $N$}

A titre d'exemple, le niveau de la capacité d'analyse associée à une activité Ai est dépendante à la fois de la proximité de cette activité par rapport à l'aspect analyse et de la complexité de l'objet sur lequel porte l'activité. L'hypothèse d'inférence est que l'effort d'analyse mis en œuvre dans l'activité (on parlera de la signature de la compétence sur l'axe analyse) est le plus élevé quand la valeur de la proximité par rapport à l'aspect analyse est maximale. Cet effort est d'autant plus élevé que cette activité porte sur un objet complexe. Nous avons retenu une échelle sur cinq niveaux de maîtrise. 
Le tableau 3 suivant montre l'exemple de constitution des règles d'inférences pour la signature de la compétence sur l'axe analyse.

\begin{tabular}{|l|l|c|}
\hline \multicolumn{2}{|c|}{ PREMISSES } & CONCLUSION \\
\hline Proximité Analyse & \multicolumn{1}{|c|}{ Complexité objet } & Niveau capacité d'analyse \\
\hline \multirow{3}{*}{ Différente } & Simple & N. 1 \\
\cline { 2 - 3 } & Relativement complexe & N. . \\
\cline { 2 - 3 } & Complexe & N. 2 \\
\hline \multirow{3}{*}{ Relativement Proche } & Simple & N. 2 \\
\cline { 2 - 3 } & Relativement complexe & N. 3 \\
\cline { 2 - 3 } & Complexe & N.4 \\
\hline \multirow{3}{*}{ Proche } & Simple & N. 4 \\
\cline { 2 - 3 } & Relativement complexe & N.5 \\
\cline { 2 - 3 } & Complexe & N.5 \\
\hline
\end{tabular}

Tableau 3. Règles d'inférences pour la capacité d'analyse

Les résultats des règles d'inférences seront obtenus en utilisant un ensemble d'opérateurs T-norme et S-norme, ils prennent la forme de fonction d'appartenance. Pour notre approche, nous avons opté pour la méthode la plus fréquente, celle du Max-Min, dite aussi méthode de Mamdani. Cette méthode nous semble appropriée à notre besoin qui consiste à trouver la valeur minimale de la compétence qui a permis d'arriver au résultat validé et satisfaisant de l'activité.

Cette méthode associe aux opérateurs "ET" et "ALORS", l'opération "min" et à l'opérateur "OU" entre les différentes règles, l'opération "max" afin de trouver la valeur minimale de la sortie (dans notre cas le niveau de compétence) garantissant le résultat décrit par l'ensemble des règles (ici c'est la combinaison situation compétence).

\subsection{Défuzzification}

Le résultat du moteur d'inférence est obtenu sous une forme floue (fonction d'appartenance). L'étape de défuzzification est l'étape inverse permettant le passage de la forme floue vers la valeur réelle. La méthode la plus utilisée est celle du centre de gravité qui consiste à calculer à l'aide de l'équation suivante, la valeur du centre de gravité $X^{*}$ de la surface résultante de l'instanciation du système d'inférence. La projection de cette valeur sur l'axe des abscisses donnera la valeur estimée de la note du composant de la compétence considérée. 


$$
X^{*}=\frac{\left.\int X_{x} \mu_{x}\left(X_{x}\right) d X_{x}\right)}{\int \mu_{x}\left(X_{x}\right) d X_{x}}
$$

\section{Exemple d'application}

Afin de tester le principe de solution proposé, nous avons appliqué notre approche sur un cas industriel. L'exemple traité concerne un projet de conception d'un nouveau meuble TV destiné aux écrans LCD. Ce projet a été réalisé dans l'entreprise de conception et fabrication de meubles, "Parisot meubles", par deux étudiants concepteurs dans le cadre de leur projet de fin d'études. Le but du projet est à la fois industriel et pédagogique. Les étudiants ont été immergés dans les services création et conception technique et ont eu ainsi une vision transversale d'un projet de conception de produits nouveaux (de l'étude du besoin à la définition technique du produit).

Les propositions des étudiants ont été approuvées, phase par phase, par les responsables techniques de l'entreprise. Cela signifie selon leur tuteur industriel (responsable du service conception), que les étudiants ont acquis, à la fin de leur expérience, des compétences de concepteurs.

\subsection{Données de traçabilité}

Le projet de conception a été structuré globalement selon trois processus directs (étude marketing, conception de principe, conception technique), trois processus "support" (logistique, prototypage, industrialisation) et un processus "manager" (validation). Nous avons effectué une traçabilité de ce projet de conception à partir de fiches que nous avons élaborées et nous avons structuré les connaissances selon le modèle générique de la situation, présenté précédemment.

Le processus de conception technique regroupe les deux étapes classiques de conception d'ensemble et de conception de détail. Il a pour objectif de "définir la solution technique" en réalisant un dossier du produit (plans ...) avec suffisamment de détails pour réaliser le prototype. Ce processus comporte six tâches de conception avec une itération de la tâche "réaliser les plans".

Considérons l'activité associée à la réalisation de cette tâche. La solution proposée ayant été retenue et lancée en industrialisation et les contraintes de réalisation ayant été respectées, la compétence correspondante a été validée par le responsable du service conception.

Le tableau 4 présente les différentes actions identifiées au cours de cette activité. Il regroupe aussi, selon leurs rôles respectifs, les différentes entités qui ont participé à la réalisation de cette activité. 


\begin{tabular}{|c|c|c|c|}
\hline Actions (AIII-3.x) & Objet & Support & Contraintes \\
\hline $\begin{array}{l}\text { 1- Modéliser la géométrie du meuble } \\
\text { 2- Rechercher avec un logiciel les } \\
\text { différentes combinaisons de } \\
\text { paramètres du tube support } \\
\text { 3- Evaluer } \\
\text { 4- Choisir une quincaillerie adaptée } \\
\text { à la solution tube en fonction des } \\
\text { paramètres trouvés précédemment } \\
\text { 5- Faire les cotations } \\
\text { correspondantes } \\
\text { 6- Choisir les composants adéquats } \\
\text { dans la quincaillerie } \\
\text { 7- Intégrer dans le modèle du meuble } \\
\text { 8- Identifier les points de contact } \\
\text { avec les nouveaux composants } \\
\text { 9- Faire les plans de cotations finales }\end{array}$ & Plans & $\begin{array}{l}\text { - Logiciel } \\
\text { CAO } \\
\text { - Manuel } \\
\text { références } \\
\text { quincaillerie } \\
\\
\text { - Logiciel } \\
\text { d'optimisation } \\
\text { du débit de } \\
\text { matière }\end{array}$ & $\begin{array}{l}\text { - Contrainte de } \\
\text { fabrication : } \\
\text { éviter les } \\
\text { petites pièces } \\
\text { et les panneaux } \\
>28 \mathrm{~mm} \\
\text { - Contrainte : } \\
\text { taux de chute } \\
<5 \% \\
\text { - Contrainte : } \\
\text { résistance du } \\
\text { meuble à une } \\
\text { charge }=70 \mathrm{~kg}\end{array}$ \\
\hline
\end{tabular}

Tableau 4. Description de l'activité "réaliser les plans"

La figure 7 présente le diagramme d'objet qui représente la structure du plan d'actions associé à l'activité "réaliser les plans". Il décrit l'enchaînement des actions élémentaires, le type de chacune de ces actions, les entités traitées par chaque action ainsi que la nature de ce traitement (création, modification, suppression). Ce modèle nous renseigne aussi sur l'occurrence de cinq entités interactionnelles transactionnelles : (deux E.I. "communication", deux E.I. "coopération par échange d'information" et une E.I. "négociation").

Le nombre total des actions dans le plan d'actions de l'activité "réaliser les plans" est de 9 , réparties comme suit :

- Le nombre d'actions de type traitement/exécution est 4

- Le nombre d'actions de type recherche/génération est 2

- Le nombre d'actions de type choix/décision est 2

- Le nombre d'actions de type mesure/évaluation est 1 


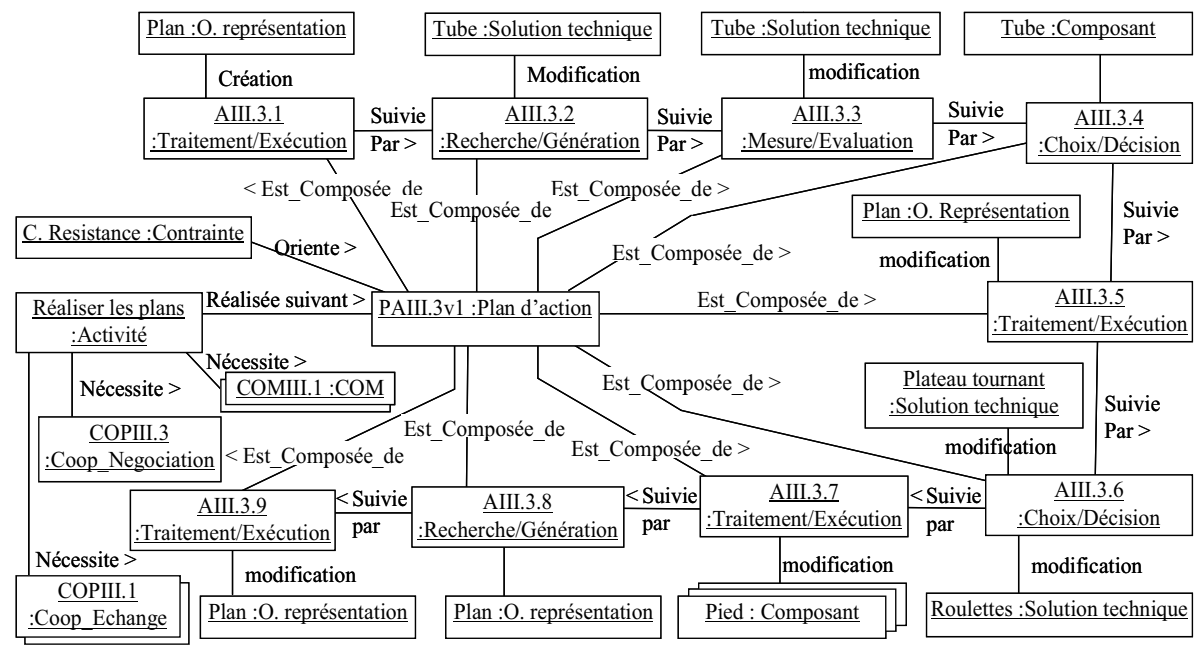

Figure 7. Plan d'actions final de l'activité "réaliser les plans"

\subsection{Résultats de la caractérisation des compétences}

L'application de la méthode a pour objectif de déterminer les caractéristiques des compétences considérées comme acquises et validées. Nous présentons ici les résultats de l'activité "réaliser les plans" du processus de "conception technique".

\subsubsection{Estimation du niveau de maîtrise de la capacité d'analyse}

Pour déterminer la signature de la compétence sur l'axe analyse, nous avons besoin des valeurs des deux variables caractéristiques: "proximité/analyse" et "complexité objet".

La valeur de la variable "proximité/analyse" est obtenue par instanciation de l'équation 2. La valeur est :

$$
\text { Prox_Analyse }=0.45=45 \%
$$

D'un autre coté, la tâche de réalisation des plans consiste principalement à fixer la quincaillerie qui va composer le meuble et à préciser la forme définitive du meuble et ses dimensions.

La complexité de l'objet est calculée en fonction des proportions de manipulation des différentes vues du produit pendant chaque action élémentaire. Au cours de l'activité étudiée, les quatre vues du produit ont été abordées, avec une dominance pour la manipulation des vues structurelle et paramétrique : la vue structurelle pour la définition de la quincaillerie et les points d'assemblage ; la vue paramétrique pour 
les cotations du meuble et des points d'assemblage. La vue comportementale n'a pas été beaucoup sollicitée dans cette activité sauf pour le calcul de l'épaisseur de la table supérieure du meuble. Une revue des différentes fonctions du meuble a été effectuée dans le choix de la quincaillerie mais aucune modification n'a été faite sur ces fonctions. La fréquence de manipulation de chaque vue a été estimée respectivement : $5 \%, 5 \%, 40 \%$ et $50 \%$.

En reprenant l'équation 4, l'expression de la variable "Compl_objet" est donc :

$$
\text { Compl_Objet }=(1 \times(5)+0.70 \times(5)+0,60 \times(40)+0.20 \times(50)) /(100)
$$

$$
\text { Compl_Objet }=0.42=42 \% \text {. }
$$

La projection des valeurs des variables "prox_Analyse" et "Compl_Objet" sur le système de traitement flou fait activer deux règles d'inférences (figure 8). Le résultat du calcul après l'étape de défuzzification donne la valeur 4,7 /10 de la signature sur l'axe analyse.
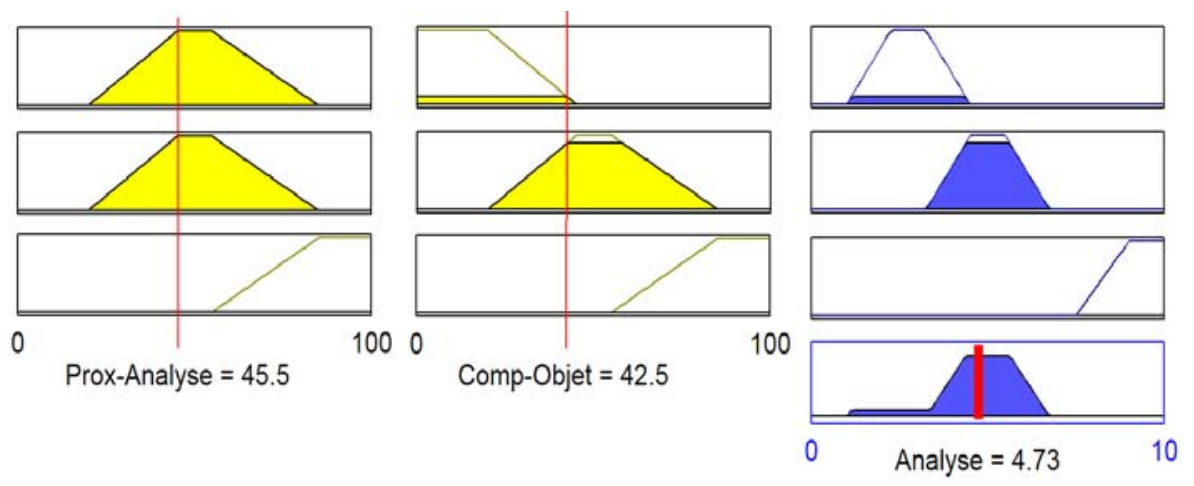

Figure 8. Exemple du mécanisme d'inférences

\subsubsection{Estimation du niveau de maîtrise de la capacité relationnelle}

La signature sur l'axe relationnel fait intervenir les variables "type de transaction" et "nombre de participants".

La valeur du type de transaction est obtenue par instanciation de l'équation 3. La valeur finale obtenue est :

Type_Transaction $=0.44=44 \%$

La valeur du nombre de participants est obtenue par instanciation de l'équation 6. Dans notre cas, les entités interactionnelles transactionnelles ont fait intervenir six 
participants ( 0 de statut I (descendant), 2 de statut II (ascendant) et 4 de statut III (sans hiérarchie)) :

Nbre_Participants $=0.36=36 \%$

La projection des valeurs de Type_Transaction et Nbre_Participants fait appel aux règles 4 et $5 \mathrm{du}$ tableau 2. La valeur de la signature sur l'axe relationnel est égale à : $4.6 / 10$.

\subsubsection{Signature finale de la compétence}

De la même manière, nous effectuons le calcul des niveaux de maîtrise des capacités décisionnelles et organisationnelles. Les valeurs estimées pour notre exemple sont représentées par la signature donnée sur la figure 9. Les cercles permettent de faciliter la lecture pour des appréciations qualitatives.

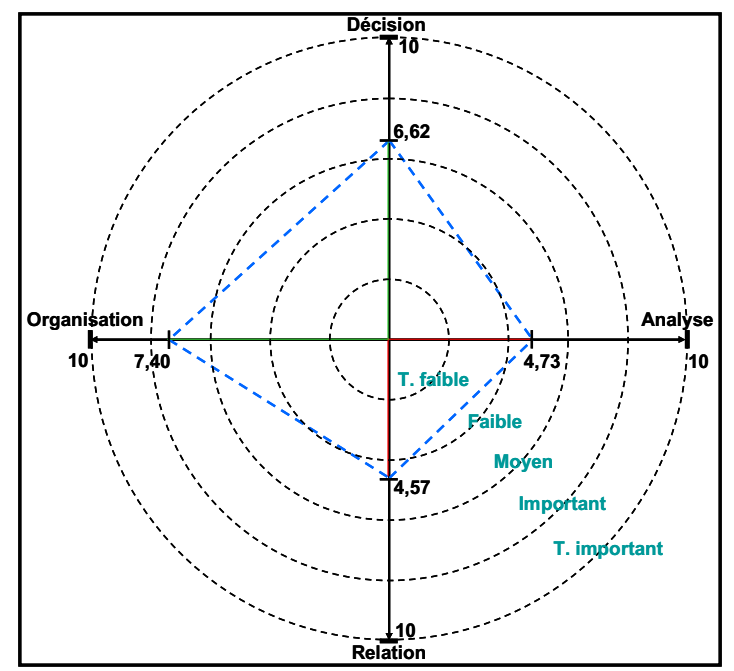

Figure 9. Signature de la compétence associée à l'activité "réaliser les plans"

Cette signature signifie que la réalisation de l'activité "réaliser les plans" a nécessité une capacité d'analyse et une capacité relationnelle dans la moyenne, une capacité de décision juste au dessus de la moyenne et une capacité d'organisation importante. Confrontées à l'avis du responsable du service conception (et du tuteur pédagogique), ces valeurs sont satisfaisantes au vu de la mission réalisée. Cela signifie aussi qu'on peut reconnaître à l'acteur qui a réalisé cette activité, le "potentiel" de mettre en œuvre un niveau semblable de capacités dans d'autres missions du même type. 
Cependant, pour d'autres types de mission, il est possible que l'acteur soit capable de mettre en œuvre des niveaux supérieurs. Dit autrement, l'interprétation de ces valeurs doit être une interprétation relative au même type de mission et de situation, et non comme une reconnaissance générique d'une capacité de l'acteur pour tout type de mission.

\subsubsection{Estimation des niveaux de maîtrise des connaissances techniques}

Pour la définition des niveaux de maîtrise des connaissances techniques, nous avons besoin des valeurs des variables caractéristiques de la proximité par rapport à l'aspect exécution ("Proximité/exécution") et de la "fréquence d'interactions" avec les entités concernées par l'activité. L'objet principal de l'activité correspond aux plans de cotations d'un meuble TV. Les acteurs ont utilisé trois supports : un logiciel $\mathrm{CAO}$, un logiciel spécifique à l'entreprise et le manuel de références de quincaillerie. Les fréquences d'interactions avec ces entités support sont estimées à $75 \%, 40 \%$ et $25 \%$ respectivement (la somme est différente de $100 \%$ à cause des utilisations simultanées). La fréquence d'interactions avec les plans est de $80 \%$ (durant l'interaction avec l'outil CAO).

La valeur de proximité par rapport à l'aspect exécution est obtenue par spécification et instanciation de l'équation 1 en donnant une pondération maximale aux actions de type "traitement/exécution". L'expression résultante est :

Prox_Exécution $=(1 \mathrm{x}(4)+0.50 \mathrm{x}(1)+0.30 \mathrm{x}(2)+0.15 \mathrm{x}(0)+0.15 \mathrm{x}(2)) / 9$.

Prox_Exécution $=0.60=60 \%$.

Les valeurs estimées pour les niveaux de maitrise des connaissances techniques dans l'activité après étape de traitement flou sont données dans le tableau 4 :

\begin{tabular}{|l|c|l|}
\hline \multicolumn{1}{|c|}{ Connaissances : Connaitre le(s) ... } & Notes/10 & Commentaire \\
\hline Logiciel CAO & 5,7 & Transposition \\
\hline Logiciel de traitement des solutions techniques & 4.4 & Application \\
\hline Appellations techniques du manuel de quincaillerie & 3.8 & Application \\
\hline Principes, règles, méthodes de construction d'un plan & 6.0 & Transposition \\
\hline
\end{tabular}

Tableau 4. Niveaux des connaissances associées à l'activité "réaliser les plans"

La présentation de ces résultats au responsable du service conception a permis de valider un rapprochement entre notre signature des compétences et les caractéristiques des compétences requises pour ce type d'activités. 


\subsection{Remarque sur l'interprétation des résultats :}

Le résultat de l'approche ne doit en aucun cas être vu comme une reconnaissance absolue de traits de personnalité ou de capacités cognitives propres à un individu. Ce résultat se résume plutôt comme une évaluation relative à une mission particulière : il s'agit de reconnaître à un acteur qu'il a mobilisé avec succès, dans le cadre de son activité, les composants de la compétence concernée. Par rapport au référentiel de compétences, ceci se traduit par une évaluation qualitative de la compétence déployée par un acteur durant l'activité en fonction des éléments tangibles de la situation.

Comme une compétence industrielle se rapporte à un type de missions (par exemple, "réaliser les plans d'un meuble") réalisées dans une classe de situations semblables (par exemple, avec les mêmes supports et managers), un groupe d'experts devra utiliser les résultats de la caractérisation pour interpréter la réussite d'une mission particulière et estimer s'il est possible d'inférer que l'acteur est potentiellement compétent pour un type de missions (généralisation).

Devant affecter une mission à un acteur, les managers pourront identifier parmi les membres de l'entreprise, ceux qui ont prouvé avoir mobilisé des compétences semblables pour un même type de mission (classes de situations similaires).

\section{Conclusion}

Nous avons proposé dans cet article une nouvelle démarche pour aider à caractériser les compétences de l'entreprise. Les résultats de cette démarche peuvent être exploités, en particulier, pour des problématiques d'affectation. Son principal avantage est son articulation avec les notions de traçabilité et de capitalisation des connaissances. L'intérêt d'une telle articulation est d'améliorer le processus de mis à jour de référentiels en se basant non pas sur les estimations subjectives d'un acteur humain (souvent, le manager) mais sur une évaluation automatique de données réelles obtenues à partir de traces capitalisées tangibles. Ces données serviront de base de discussion pour la reconnaissance des compétences, ce qui permettra d'améliorer la précision et la fiabilité des informations contenues dans les référentiels des entreprises et d'ajouter un caractère "dynamique" à ces référentiels afin qu'ils prennent en compte, par des mises à jour régulières, les développements technologiques et les évolutions permanentes sur les méthodes de travail, sur les connaissances techniques, etc. Le "principe de solution" proposé ouvre la porte à de nouvelles applications pour les systèmes de capitalisation de connaissances, autres que celles de mémoire projet pour la réutilisation et l'aide à la conception technique.

Nous précisons que la méthode, bien que basée sur un modèle théorique et cognitif de la compétence, n'a pas la prétention d'identifier des traits de personnalité, ou de caractériser des processus cognitifs dans l'absolu. Il s'agit plutôt 
de caractériser les capacités déployées durant l'activité en fonction des éléments tangibles de la situation.

Notre première application en milieu industriel nous a permis de confirmer la validité de ce principe de solution pour résoudre ce type de problématique. Le tableau 2 ainsi que les relations entre "situation et compétence" ont donné lieu à discussion avec les experts de la conception pour expliciter ce qu'est une compétence (quels composants), quelles sont les caractéristiques objectivables des situations concernées et comment les relier aux composants de la compétence. La caractérisation de compétences, au-delà de la notation, a aidé à prendre conscience et à formaliser des connaissances manipulées, des capacités opératoires et des difficultés liées à leur acquisition et mise en œuvre.

Un référentiel de compétences n'est pas une fin en soi. Les discussions et négociations sociales qui doivent s'engager sur la nature et les conditions de l'activité des acteurs sont certainement des plus importantes dans un premier temps mais par la suite, un référentiel de compétences fiable est nécessaire pour un bon pilotage des activités de l'entreprise.

D'autres applications sont à prévoir pour confirmer la maturité de nos concepts. Selon le vocabulaire et les pratiques de l'entreprise, les composants de la compétence et les variables que nous avons proposés devront être adaptés après discussion avec les managers et les acteurs concernés, sans remettre en cause le cour de la démarche.

Cependant, certaines limites de ce travail peuvent être liées à la complexité de la phase d'extraction des informations dans le cas où la granularité demandée est trop élevée. L'efficacité du module de traçabilité repose sur la motivation du concepteur à renseigner les informations décrivant son activité. Certains facteurs peuvent le démotiver : la durée de la saisie, la crainte que ces informations soient utilisées pour le contrôler, l'incompréhension de la modélisation. Une façon d'inciter ce concepteur à adopter la démarche serait d'intégrer les modèles dans les systèmes informatiques d'utilisation quotidienne. Ces outils peuvent aussi lui offrir l'opportunité de pouvoir partager son expérience avec d'autres, d'identifier les meilleures pratiques pour améliorer sa performance et de prouver qu'il est compétent (exigence précisée dans la norme ISO 9000 version 2000, partie "management des ressources humaines"). Une autre limite de la démarche, que l'on retrouve souvent dans des approches qualitatives d'aide à la décision, provient du choix des pondérations. Ce choix pourrait être enrichi par confrontation entre différents experts et éventuellement par des mécanismes d'apprentissage de type "réseaux de neurones".

Les prochaines étapes envisagées pour l'amélioration de la démarche sont, d'un coté, de généraliser l'approche sur tout l'historique des activités similaires et d'un autre coté, d'intégrer la méthode dans un système plus global de pilotage des compétences. L'utilisation d'un système de raisonnement à partir de cas pourrait être bénéfique (Bonjour, 2008) pour classer les compétences acquises comme des situations-problèmes ("cas résolu") et procéder à l'appariement avec une compétence 
requise ("nouveau cas"), la mise en œuvre pouvant nécessiter une adaptation des composants de la compétence ("cas révisé").

\section{Bibliographie}

Acuna S.T., Juristo N., « Assigning people to roles in software projects », Software Practice and Experience, Vol. 34,2004, p. 675-696.

Ahmad S., Schroeder R.G., "The impact of human resource management practices on operational performance: recognizing country and industry differences », Journal of Operational Management, Vol. 21, 2003, p. 19-43.

Araujo R.M., Santoro F.M., Brézillon P., Borges M., Rosa M., «Context models for managing collaborative software development knowledge », Proc. of the KI Workshop on Modeling and Retrieval of Context, http://CEUR-WS.org/ Vol-114, Germany, 2004.

Athey T.R., Orth M.S., "Emerging Competency Methods for the Future », Human Resource Management, New York, Vol. 38, ํ3, 1999, p. 215-225.

Belkadi F., Contribution au pilotage des compétences dans les activités de conception : de la modélisation des situations à la caractérisation des compétences. Thèse de doctorat, Université de Franche Comté, 2006.

Belkadi F., Bonjour E., Dulmet M., «Competency characterisation by means of work situation modelling », Computers in industry, Vol 58, N 2, 2007, p. 164-178.

Belkadi F., Bonjour E., Dulmet M., « Proposition of a Situation Model in View to Improve collaborative design ", INCOM'04, $11^{\text {th }}$ IFAC Symposium on Information Control Problems in Manufacturing, Brazil 5-7 April 2004.

Bennour M., Crestani D., «Using competencies in performance estimation: from the activity to the process ", Computers in Industry, Vol. 58, N² 2, 2007, p. 151-163.

Berio G., Harzallah M., « Knowledge Management for Competence Management », Journal of Universal Knowledge Management, vol. 0, No. 1, 2005, p. 21-28.

Bonjour E., Dulmet M., Lhote F., « An internal modeling of competency, based on a systemic approach, with socio-technical systems management in view », Proc. of IEEE International Conference on Systems, Man and Cybernetics, Hammamet, Tunisie, 6-9 octobre 2002.

Bonjour E., Du retour d'expériences au développement des compétences, in J. Renaud, E. Bonjour, B. Chebel-Morello, B. Fuchs, N. Matta : Retour et capitalisation d'expériences, (Chapitre 5), AFNOR, 2008, p. 155-198.

Borges M., Brézillon P., Pino J-A, and Pomerol J.-Ch., « Groupware system design and the context concept ». Lecture Notes in Computer sciences, $\mathrm{N}^{\circ} 3168$, Springer-Verlag, 2005, p. 45-54.

Boucher X., Bonjour E., Grabot B., « Formalisation and use of competencies for industrial performance optimisation: a survey », Computers in Industry, Vol. 58, $\mathrm{N}^{\circ} 2,2007$, p. 98117.

Boucher X., Burlat P., "Vers l'intégration des compétences dans le pilotage des performances de l'entreprise ", Journal Européen des Systèmes Automatisés, Vol. 37, $\mathrm{N}^{\circ} 3,2003$, p. 363-390. 
Bouchon-Meunier B., Marsala C., Logique floue, principes, aide à la décision. Ed. Hermès 2003.

Canos L., Liern V., « Some fuzzy models for human resource management », Int. Journal of Technology, Policy and Management, Vol. 4, N 4, 2004, p. 291-308.

Cavestro W., Durieux C., Monchatre S., Travail et reconnaissance des compétences, Paris, Economica, série Connaissance de la Gestion, 2007.

Centre national de la fonction publique territoriale, direction du développement des compétences territoriales Référentiels d'emploi - activités - compétences, Guide méthodologique éd. CNFPT Paris, juin 2001. ISBN : 2- 84143-190 -8.

Chandrasekaran B., « Design problem solving: A task analysis », AI Magazine, Vol. 11, 1990, p. 59-71.

De Korvin A., Shipley F., Kleyle R., « Utilizing fuzzy compatibility of skill sets for team selection in multi-phase projects ", Journal of Engineering Technology Management, Vol 19, 2002, p. 307-319.

De Terssac G., "Le travail : un objet commun », 2ème journée recherche et ergonomie, Toulouse, Février 1998, p. 90-99.

Dejoux C., Les compétences au cour des performances, Ed. d'organisation, 2001.

Dronkov D., Reinfrank M., Hellendom H., An Introduction to Fuzzy Control, SpringerVerlag, London, 1993.

Dubois D., Foulloy L., Galichet S., Prade H., " Two different views of approximate reasoning ", Proc. of the 7th Int. Fuzzy Systems Association World Congress (IFSA'97), Prague, Czech Republic, Vol. 1, June 97, p. 238-242.

Dulmet M., Bonjour E., « Vers un méta-modèle de pilotage du système de compétences de

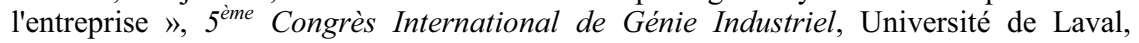
Québec, Canada, 26-29 octobre 2003.

Engeström Y., Learning by expanding: an activity-theoretical approach to developmental research, Helsinki: Orienta-Kosultit Oy, Finland, 1987.

Ermine J.L, Les systèmes de connaissances, Eds. Hermès, 1996.

Faucher R., Petterson N., Naji A., Notions de compétence individuelle et de compétence collective : répertoire de définitions, document de travail No. 0203-04, École des sciences de la gestion Université du Québec à Montréal, 2003.

Fitzpatrick E.L., Askin R.G., « Forming effective worker team with multi-functional skill requirement », Computer and Industrial Engineering, Vol. 48, 2005, p. 593-608.

Ganascia J., Les sciences cognitives, éd. Flammarion, 1996.

Gronau N., Froeming J., Schmid S., Ruessbuelt U., « Approach for requirement oriented team building in industrial processes », Computers in Industry, Vol. 58, N² 2, 2007, p. 179-187.

Grundstein M., From capitalizing on Company Knowledge to Knowledge Management, In Knowledge Management, Classic and Contemporary Works, chapter 12, the MIT Press, Cambridge, Massachusetts, , 2001, p. 261-287.

Hadj-Hamou K., Caillaud E., « Cooperative Design: a Framework for Competency Based Approach ", 5th Int. Conf. on Integrated Design and Manufacturing in Mechanical 
Engineering, IDMME’04, Université de Bath, 5-7 avril 2004.

Harzallah M, Berio G, Vernadat F., « Analysis and Modeling of Individual Competencies: Towards better management of human resources ", IEEE Transactions on Systems, Man and Cybernetics, Part A, Vol. 36, Jan. 2006, p. 187-207.

Harzallah M., Modélisation des aspects organisationnels et des compétences pour la réorganisation d'entreprise industrielles, thèse de doctorat, Université de Metz, 2000.

Herrera F., Herrera-Viedma E., Verdegay J.L., A linguistic Decision Process in Group Decision Making, Technical report, DECSAI-94102, Granada University, Spain 1994.

Houé R., Grabot B., Geneste L., « Competence management for business integration », 12th IFAC Symposium on INformation COntrol problems in Manufacturing, INCOM, IFAC/IEEE/IFIP, Saint-Etienne, May 2006.

Houssin R., Bernard A., Martin P., Ris G., Cherrier F., « Information system based on a working situation model for a new design approach in concurrent engineering », Journal of Engineering Design, Vol. 17, № 1, p. 35-54, 2006.

Jolis N., Piloter les compétences, de la logique de poste à l'atout compétence, éd. d'organisation, Paris, 1997.

Jussupova-Mariethoz Y., Probst R., "Business Concepts Ontology for an enterprise performance and competences monitoring", Computers in industry, Vol. 58, N ${ }^{\circ} 2,2007$, p. 118-129.

Kirsch-Pinheiro M., Gensel J., and Martin H., «Representing Context for an Adaptative Awareness Mechanism », Lecture Notes in Computer sciences, $\mathrm{N}^{\circ} 3198$, SpringerVerlag, 2004, p. 339-348.

Klein AL, «Validity and Reliability for Competency-based systems: Reducing litigation Risks ", Compensation \& Benefits Review, Vol. 28, № 4, 1996, p. 31-37.

Kofod-Petersen A., Cassens J., « Using Activity Theory to Model Context Awareness », Lecture Notes on Artificial Intelligence, Vol. 3946, 2006, p. 1-17.

Le Boterf G., Construire les compétences individuelles et collectives, Ed. d'organisation, Paris, 2000.

Le Boterf G., Ingénierie et évaluation des compétences, Ed. d'Organisation, 2002.

Le Moigne J.L., La modélisation des systèmes complexes, AFCET Systèmes, Ed. Dunod, 1990.

Lesage C., Evaluation du risque d'audit : proposition d'un modèle linguistique, Cahier de Recherche du CEREG, N9713, 1997

Levy-Leboyer C., La gestion des compétences, Ed. d'organisation, 1996.

Ley T., Organizational Competency Management: A Competence Management Approach, Shaker Verglas, 2006.

Livian Y.F., Organisation, théories et pratiques, Ed. Dunod, 1998.

Lucia A.D., Lepsinger R., The art and science of competency models: Pinpointing critical success factors in Organizations, Jossey-Bass, 1999.

Malvache P., Prieur P., « Mastering Corporate Experience with the REX Method », In J.P. Barthès Ed., Proceedings of ISMICK'93, Compiègne, October 1993, p. 33-41.

Matta N., Corby O. Ribiere M., Méthodes de capitalisation de mémoire de projet, INRIA, 
Rapport de recherche $\mathrm{N}^{\circ} 3819,1999$.

MEDEF, L'influence des contextes nationaux sur le management par les compétences, Cahiers du MEDEF, Objectif compétences, des pratiques européennes innovantes, France 2002.

Micaëlli J.P., Forest J., Artificialisme, Introduction à une théorie de la conception, collection Epistémologie, INSA de Lyon, 2003.

Michel S., Ledru M., Capital compétence dans l'entreprise : une approche cognitive, éd. ESF, 1991.

Midler C., "Evolution des modèles d'organisation et régulation économiques de la conception », Annales des Mines, Février 1997.

Mitrani A., Dalziel M., Bernard A., Des compétences et des hommes : le management des ressources humaines en Europe, Éd. d'Organisation, 1992.

Nonaka I., Takeuchi H., The knowledge-Creating Company : How Japanese Companies Create the Dynamics of Innovation, Oxford University, 1995.

Oget D., Sonntag M., « Aspects cognitifs et conatifs de la compétence collective », 4ème congrès international de Génie Industriel, Marseille, 12-15 juin 2001.

Pahl G., Beitz W., Engineering Design: a Systematic Approach, Springer-Verlag, 1996.

Parsons T., Toward a General Theory of Action, In: Parsons T., Shils E.A. (Eds.), Towards a General Theory of Action: Theoretical Foundations for the Social Sciences. Harper \& Row, New York, NY, 1965.

Pépiot G., Méthodologie des entreprises sur la base des compétences, thèse de doctorat en génie mécanique, EPFL de Lausanne, 2005.

Pierre C., Jouvenot C., La reconnaissance au cour des démarches compétence, enjeux et repères pour l'action, Ed. ANACT, Lyon, juillet 2007.

Piaget J., L'équilibration des structures cognitives, PUF, 1975.

Prahalad C.K., Hamel G., "The core competence of the corporation », Harvard Business review, may-june 1990, p. 79-91.

Prax J.Y., Le guide du Knowledge Management - Concepts et pratiques de la gestion des connaissances, éd. Dunod, 2000.

Rasmussen J. Information processing \& human machine interaction An approach to cognitive engineering, éd. Sage 1986.

Rault-Jacquot V., Contribution à la valorisation du patrimoine technologique de l'entreprise: Proposition d'une approche de l'inventaire et de l'évaluation des compétences, thèse de doctorat en génie industriel, I.N.P. Loraine, 1993.

Sardas J-C., "Ingénierie intégrée et mutation des métiers de la conception », Annales des Mines, 1997, p. 41-48.

Searle J., La construction de la réalité sociale, Jacob, Paris, 1998.

Skyrme D., « The Knowledge Asset, Management Insight », David Skyrme Associates, N ${ }^{\circ}$ 11,1994

Spencer L.M., Spencer S.M., Definition of competency, In Competence at Work, Models for Superior Performance, Chapter 2, John Wiley and Sons inc, 1993, p. 9-16. 
Stenlund K.L, Hörte S.A., « Competence accounting - methods for measuring and valuing key competencies » European Operations Management Association VI International Annual Conference "Managing Operations Networks", Italy, 7-8 June 99.

Studer R, Benjamins V.R, Fensel D., « Knowledge Engineering: Principles and methods », Data and Knowledge Engineering, Vol. 25, 1998, p. 161-197.

Tseng T-L., Huang C-C., Chu H-W., Gg R-R., « Novel approach to multi-functional project team formation », International Journal of Project Team, Vol. 22, 2004, p. 147-159.

Vaudelin, J-P., O. Devise, «L'intégration des compétences en vue de l'amélioration de la performance industrielle d'une unité de production ", Revue Française de Gestion Industrielle, $\mathrm{Vol} 24,2005, \mathrm{~N}^{\circ} 1$, p. 7-17.

Vergnaud G., Au fond de l'action, la conceptualisation In J.M Barbier, Savoirs théoriques et savoirs d'action, Education et formation, PUF, Paris, p. 275-292, 1998.

Vidal-Gomel C., Samurçay R., «Qualitative analyses of accidents and incidents to identify competencies », Safety Science, Vol. 40, 2002, p. 479-500.

Vygotsky L., Mind in Society, Cambridge, MA: Harvard University Press, 1978.

Zadeh L., «Fuzzy Set », Information and Control, Vol. 8, 1965, p. 338-353.

Zimmermann H.J, Fuzzy set theory, and its applications, Kluwer Academic Publishers, Dordrecht, 4th Ed., 2001. 